\title{
Radiological Survey in Soil of South America
}

\author{
María Luciana Montes and Judith Desimoni \\ Departamento de Física, Facultad de Ciencias Exactas, Universidad Nacional de La Plata \\ Instituto de Física La Plata - CONICET, \\ Argentina
}

\section{Introduction}

When the Earth was formed, the crust and consequently the soil and water were conformed by a wide variety of chemical elements with different concentrations; being some of these radioactives. There are different activity levels of natural radionuclides, as those of the $238 \mathrm{U}$ and ${ }^{232} \mathrm{Th}$ decay chains, ${ }^{40} \mathrm{~K},{ }^{7} \mathrm{Be}$ and ${ }^{14} \mathrm{C}$, etc. along the planet [Cooper et al., 2003]. Among the 80 nuclides found in the environment, the more relevant concerning the radiobiological significance are ${ }^{40} \mathrm{~K}$, and the nuclides belonging to the ${ }^{238} \mathrm{U}$ and ${ }^{232} \mathrm{Th}$ decay chains. The human activities can strongly modify the natural concentrations due to the presence of residues or accumulation of elements caused by the release of effluents to the environment. In the 60's the nuclear power production and nuclear weapon testing discharge to the environment anthropogenic nuclides. In particular, the Southern Hemisphere was mainly polluted by the debris originated in the South Pacific and middle Atlantic nuclear weapon tests [UNSCEAR, 2008]. Along with the class of anthropogenic gamma emitter nuclides releases, the ${ }^{137} \mathrm{Cs}$ is the most prominent isotope in the Earth crust originated by fission process. It is considered as one of the hazardous environmental contaminant due to the contribution to the external irradiation exposure and its incorporation to the human food chain [Singh et al., 2009].

Regardless, both natural and man-made nuclides have radiobiological implication because they significantly contribute to human external radiation dose and to the internal dose by inhalation and ingestion [Cooper et al., 2003; UNSCEAR, 2008]. The United Nations Scientific Committee on the Effects of Atomic Radiation (UNSCEAR) has estimated that exposure to natural sources is approximately $98 \%$ of the total radiation dose (excluding medical exposure) [UNSCEAR, 2000; UNSCEAR, 2008]. The dose arising from natural nuclides varies worldwide depending upon factors such as height above sea level, the amount and type of radionuclides in the air, food and water, as well as the concentration of the natural nuclides in the soil and rocks, which in turn depend on the local geology of each region, etc.

The information about the presence and migration anthropogenic radionuclides is crucial to fully understand the long-term behaviour in the environment, the uptake by flora and fauna including the human food chain, as well as potential contribution to groundwater. In consequence, before assessing the radiation dose to the population, a precise knowledge of the activity of a number of radionuclides is required [UNSCEAR, 2000; 
UNSCEAR, 2008]. The mobility of the radionuclide in the ecosystem involves a number of complex mechanisms [Velasco et al., 2006; IAEA, 2010; Salbu, 2009; Cooper et al., 2003; Sawhney, 1972; Cornell, 1993; Staunton et al. , 2002; Bellenguer et al., 2008], and their transfer through the environmental compartments implies multiple interactions between the biotic and abiotic components of the ecosystem, as well as human interferences like the use of fertilizer [Tomazini da Conceic \& Bonotto, 2006] or the overexploitation of the natural resources. For the identification of these interactions it is necessary to develop and test predictive models describing the radionuclide fluxes from the environment to the man.

In South America, the soil resource is extensively used in agriculture, stockbreeding and for building materials. Baselines of natural and anthropogenic activity nuclides in several countries are not established ye, as well regulations concerning the natural and anthropogenic activity and chemical restrictions in freshwater and food accordingly to the local situations. These facts and the scattered of the activity dataset put in relevance the present review on nuclide activity determinations in soils of South America, that could be considered as the first attempt in this direction.

A systematic compilation of radionuclide activity data of soil of Argentina, Brazil, Chile, Venezuela and Uruguay are presented. Radionuclide activity data concern to the natural ${ }^{40} \mathrm{~K}, 238 \mathrm{U}$, and ${ }^{232} \mathrm{Th}$ and to the anthropogenic ${ }^{137} \mathrm{Cs}$ nuclides. These different pieces of information are put together, the quality of the environmental compartments is provided and the impact on the population is evaluated throughout the exposure dose. The migration of ${ }^{137} \mathrm{Cs}$ in soil is also analysed in the frame of different approaches [Kirchner, 1998; Schuller et al., 1997], and the transport parameters are discussed. Moreover, the caesium inventories are compared with the latitudinal UNSCEAR predictions [UNSCEAR 2000, UNSCEAR 2008].

\section{Radionuclides in the environment}

The man is continuously exposed to natural radiation since radioactive material is present in throughout nature. It occurs naturally in the soil, rocks, water, air, and vegetation. The components of the natural radioactive background are the cosmic radiation and the natural radioactivity of ground, atmosphere and water. Natural environmental radioactivity arises mainly from primordial radionuclides, such as ${ }^{40} \mathrm{~K}$ and the nuclides from the ${ }^{232} \mathrm{Th}$ and $238 \mathrm{U}$ series, which are at trace levels in all ground formations. Natural environmental radioactivity and the associated external exposure due to gamma radiation are primarily up to the geological and geographical conditions [UNSCEAR, 2000]. The specific concentrations of terrestrial environmental radiation are related to the composition of each lithologically separated area, and to the type of parental material from which the soils originate.

The high geochemical mobility of radionuclides in the environment allows them to move easily throughout the environmental matrixes. Rivers erode soil which contains radionuclides, and they reach lakes and oceans; atmospheric depositions can also occur on their surfaces; and groundwater containing some radionuclides can reach them.

Concerning the presence of artificial nuclides in the environment, after bombarding Hiroshima and Nagasaki in 1945, USA, USSR, France, England and Chine deserved to be a nuclear potency. In this frame, 543 underground and atmospheric nuclear weapon essays were carried from 1945 to 1980 in different regions of the globe. URSS, Chine and USA performed the tests in the North Hemisphere, while England and France in the South Hemisphere. The 
underground essays were the more numerous; however, the global environmental impact resulted small because the radioactive material remains in the essay area. On the contrary, the atmospheric ones delivered to the atmosphere huge amounts of radioactive detritus causing a big impact on the environment [UNSCEAR, 2008; Valkovic, 2000]. It is worth to mention that because of the atmospheric circulation, approximately the $82 \%$ of the debris remain in the hemisphere of injection [UNSCEAR, 2008; Valkovic, 2000]. The relevant nuclides originate in the essays were ${ }^{3} \mathrm{H},{ }^{14} \mathrm{C},{ }^{54} \mathrm{Mn},{ }^{55} \mathrm{Fe},{ }^{85} \mathrm{Kr},{ }^{89} \mathrm{Sr},{ }^{90} \mathrm{Sr},{ }^{95} \mathrm{Zr},{ }^{103} \mathrm{Ru},{ }^{106} \mathrm{Ru},{ }^{131} \mathrm{I},{ }^{137} \mathrm{Cs},{ }^{131} \mathrm{Ce}$ and ${ }^{144} \mathrm{Ce}$, among others [UNSCEAR, 1982]. Due to ${ }^{90} \mathrm{Sr}$ and ${ }^{137} \mathrm{Cs}$ are volatiles and have large half-life (28.6 years and 30.2 years, respectively) they are dispersed in the atmosphere, comprising the stratospheric global fallout, contributing to the residual background.

When analyzing the total annual effective dose received by human from natural sources, the dose received by the cosmic ray, terrestrial exposure, ingestion and inhalation of long-lived natural radionuclides needs consideration. Each environmental matrix, e.g. soil, air and water, has several associated pathways. These three environmental media cannot be thought as isolated and so, nuclide transfers are produced from one to the other. The different pathways exposure routes are schematized in the Fig. 1. The importance of these paths depends upon the particular radionuclide or radionuclides present in each compartment. The starting point to evaluate the people doses is to determine the nuclide concentrations in the environmental matrixes [USNCEAR, 2000; UNSCEAR, 2008].

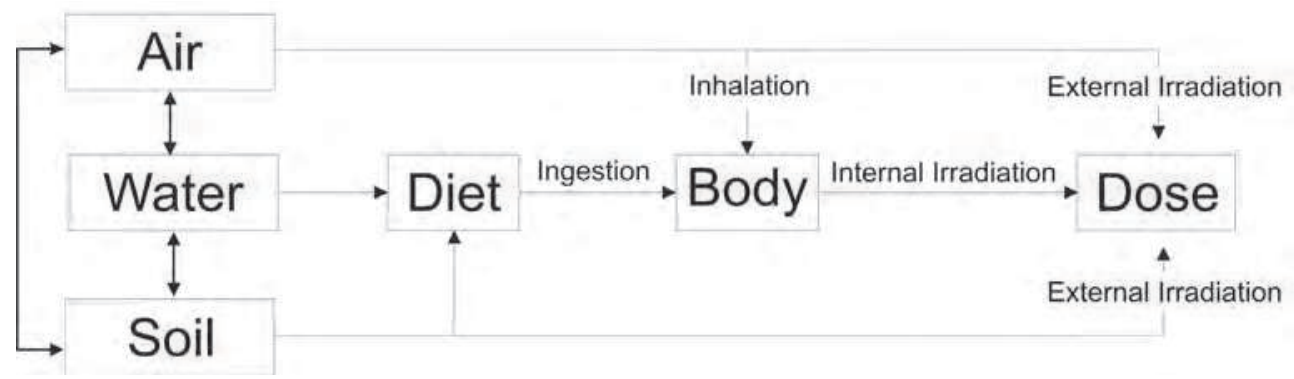

Fig. 1. Schematic terrestrial pathways of nuclide transfers and dose to humans.

\section{Monitored regions and dataset}

Two kinds of surveys have been performed, some of them deal with the determination of nuclide activity concentrations in depth, while others only reported single values of surface activity concentrations. Argentina, Brazil and Chile are the most studied countries, while there are reported a few data of Venezuela and Uruguay. The location of the monitored places, type of survey and monitored nuclides are summarized in the Table 1 . Regarding the natural nuclides in South America, the reports of UNSCEAR only account values of the activity concentration of the natural nuclide ${ }^{40} \mathrm{~K}$ for Argentina [UNSCEAR, 2000; UNSCEAR, 2008]. In San Luis Province, Argentina, two sites have been studied [Juri Ayub et al., 2008]. Recently, the first systematic studies to establish baseline activities for the naturally occurring radionuclides in unperturbed soils around La Plata city, Province of Buenos Aires, have been settled on samples taken from the surface down to a depth of $50 \mathrm{~cm}$ [Montes et al. 2010a, 2010b]. Moreover, in four superficial soils in the Ezeiza region, Argentina, the 
activities of ${ }^{40} \mathrm{~K}$ and of natural chains of ${ }^{238} \mathrm{U}$ and ${ }^{232} \mathrm{Th}$ have been determined [Montes et al. 2011]. In the Brazilian State of Rio Grande do Norte the average concentrations of 226Ra, ${ }^{232} \mathrm{Th}$ and ${ }^{40} \mathrm{~K}$ in unperturbed soils have been determined [Malanka et al., 1996]. Samples of soils were also studied in different departments of Uruguay since 2004 to determine the activity concentrations of ${ }^{40} \mathrm{~K},{ }^{226} \mathrm{Ra}$ and ${ }^{232} \mathrm{Th}$ up to $5 \mathrm{~cm}$ of depth [Odino Moure, 2010]. Regarding the anthropogenic nuclides, in Argentina ${ }^{137} \mathrm{Cs}$ reference activity profile was determined in the Pampa Ondulada region [Bujan et al., 2000, 2003] and in the central part of the country in natural and semi-natural grassland regions [Juri Ayub et al., 2007, 2008]. Beside the natural chains values, the profiles of ${ }^{137} \mathrm{Cs}$ in the region of Buenos Aires Province have been settled [Montes et al. 2010a, 2010b]. Some studies have been performed in Brazil, dealing with the determination of the activity of the ${ }^{137} \mathrm{Cs}$ globally presented on the soil because of nuclear weapon tests [Correchel et al., 2005; Handl et al., 2008]. Total inventories and depth distributions of ${ }^{137} \mathrm{Cs}$ were established in agricultural and sheep-farming regions of Chile [Schuller et al., 1997, 2002, 2004]. In Uruguay, surface soil ${ }^{137}$ Cs activity has been determined in different regions since 2004 [Odino Moure, 2010]. In Venezuela, the ${ }^{137} \mathrm{Cs}$ concentration at two different depth $(0 \mathrm{~cm}-20 \mathrm{~cm}$ and $20 \mathrm{~cm}-40 \mathrm{~cm})$ were measured [SajóBohus et al., 1999].

\subsection{Natural radionuclides}

According to the UNSCEAR [UNSCEAR, 2000], in South America only the activity concentration of ${ }^{40} \mathrm{~K}$ in unperturbed soils has been measured in Argentina (UN in Table 1 and 2), being the activity concentration range $540 \mathrm{~Bq} / \mathrm{kg}-750 \mathrm{~Bq} / \mathrm{kg}$. Later, data profiles of ${ }^{226} \mathrm{Ra}$ and ${ }^{40 \mathrm{~K}}$ of semi-natural grassland soils of the central part of the country, Province of San Luis (AS23 and AS24) have been reported down to $25 \mathrm{~cm}$ depth [Juri Ayub, 2008]. The activity concentrations of ${ }^{40} \mathrm{~K}$ were determined to vary from $720 \mathrm{~Bq} / \mathrm{kg}$ to $750 \mathrm{~Bq} / \mathrm{kg}$ very close to the upper limit of the values reported by UNSCEAR [UNSCEAR, 2000; UNSCEAR, 2008] , while $226 \mathrm{Ra}$ activities were in the range $64 \mathrm{~Bq} / \mathrm{kg}$ to $73 \mathrm{~Bq} / \mathrm{kg}$, as observed in Fig. 2. The profiles recorded down to $22.5 \mathrm{~cm}$ indicated that both nuclides activity concentrations are constant in depth (see Fig.2). Activity concentrations down to $50 \mathrm{~cm}$ of natural nuclides (238 $\mathrm{U}$ and ${ }^{232} \mathrm{Th}$ chains and ${ }^{40} \mathrm{~K}$ ) have been determined in soil samples collected from inland (AS1 and AS2) and coastal (AS3 and AS4) areas of the La Plata River, located in the North eastern region of the Province of Buenos Aires, Argentina [Montes et al., 2010a; Montes et al., 2010b]. The main observed activity resulted originated from the decay of the ${ }^{40} \mathrm{~K}$ with following in importance those of the natural $238 \mathrm{U}$ (obtained from the $226 \mathrm{Ra}$ activity) and ${ }^{232} \mathrm{Th}$ (obtained from the ${ }^{228} \mathrm{Ac},{ }^{212} \mathrm{~Pb},{ }^{212} \mathrm{Bi}$ and ${ }^{208} \mathrm{Tl}$ activities) chains, as shown in Fig. 2 . While the activity of $235 \mathrm{U}$ was, in all the cases, lower than the detection limit $\left(\mathrm{L}_{\mathrm{D}}=0.02 \mathrm{~Bq} / \mathrm{kg}\right)$, the activity values of the ${ }^{238} \mathrm{U}$ and ${ }^{232} \mathrm{Th}$ chains lay in the intervals $52 \mathrm{~Bq} / \mathrm{kg}-104 \mathrm{~Bq} / \mathrm{kg}$ and 32 $\mathrm{Bq} / \mathrm{kg}-50 \mathrm{~Bq} / \mathrm{kg}$, respectively. In the case of the $238 \mathrm{U}$, the activities resulted to some extent high when comparing with data from Uruguay [Odino Moure, 2010]. It was also observed that the coastal soils without magnetite and lower hematite relative fraction presented a higher $U$ probably related to the geological origin of the soils [Montes et al., 2010a; Montes et al., 2010b]. The ${ }^{40} \mathrm{~K}$ activity profiles were quite different when comparing the monitored soils, ranging the surface activities values from $531 \mathrm{~Bq} / \mathrm{kg}$ to $873 \mathrm{~Bq} / \mathrm{kg}$ as observed in Fig. 2. In the inland profiles, the activity increased with depth and the depletion of the activity was detected in the approximately first $20 \mathrm{~cm}$ of the inland soils. The $\mathrm{Fe}^{3+}$ relative fractions 
determined from Mössbauer spectroscopy [Vandenberghe, 1991] and the ${ }^{40} \mathrm{~K}$ distribution had quite similar behaviour. This correlation could be ascribed to the soil pedogenic and edaphic properties [Montes et al, 2010b], as well as to the presence of plant roots that use both ions as nutrients.

The other studied region of the Buenos Aires Province is located in the neighbourhood of the Centro Atómico Ezeiza [Valdés et al, 2011]. In this case the monitoring dealt with surface samples and ${ }^{238} \mathrm{U},{ }^{232} \mathrm{Th}$ and ${ }^{40} \mathrm{~K}$ activities were determined down to $10 \mathrm{~cm}$ (AS5- AS11) [Montes, 2011]. The activities, quoted in Table 2 and Fig. 3, ranged from $52 \mathrm{~Bq} / \mathrm{kg}$ to $65 \mathrm{~Bq} / \mathrm{kg}$, $24 \mathrm{~Bq} / \mathrm{kg}$ to $35 \mathrm{~Bq} / \mathrm{kg}$ and from $470 \mathrm{~Bq} / \mathrm{kg}$ to $644 \mathrm{~Bq} / \mathrm{kg}$ for ${ }^{238 \mathrm{U}},{ }^{232} \mathrm{Th}$ and ${ }^{40} \mathrm{~K}$, respectively.

In a frame of a survey program to study the environmental radioactivity in the Brazilian State of Rio Grande do Norte (BS1), the average concentrations of $226 \mathrm{Ra}(29.2 \mathrm{~Bq} / \mathrm{kg}),{ }^{232} \mathrm{Th}$ $(47.8 \mathrm{~Bq} / \mathrm{kg})$ and ${ }^{40} \mathrm{~K}(704 \mathrm{~Bq} / \mathrm{kg})$ in fifty-two soil samples down $20 \mathrm{~cm}$ in areas with homogeneous lithology of the eastern and central regions of this states were determined. These values were higher than the world average and consistent with the predominance of granites and other Precambrian igneous rocks in the region [Malanca et al., 1996].

In Uruguay, the surface (down to $5 \mathrm{~cm}$ ) ${ }^{40} \mathrm{~K}$ activity values (US1-US8) ranged from 89.9 $\mathrm{Bq} / \mathrm{kg}$ up to $1054 \mathrm{~Bq} / \mathrm{kg}$ while activity values of ${ }^{226} \mathrm{Ra}$ and $232 \mathrm{Th}$ were from $7.2 \mathrm{~Bq} / \mathrm{kg}$ to 23.2 $\mathrm{Bq} / \mathrm{kg}$ and $5.5 \mathrm{~Bq} / \mathrm{kg}$ to $75.4 \mathrm{~Bq} / \mathrm{kg}$, respectively [Odino Moure, 2010].

The data of the all determined surface activity concentrations are compiled in Table 2 and Fig. 3 together with the worldwide average data reported by the UNSCEAR [UNSCEAR, 2008]. The mean and range worldwide values have been included by completeness [UNSCEAR, 2000; UNSCEAR, 2008]. It is clear that the reported data for $238 \mathrm{U}$ for Brazil and Argentina are higher than the worldwide mean values. The observed 232Th activities of Argentina are close to the worldwide mean values, while the Brazilian ones are quite higher than the worldwide average values. Due to the scattering and the scarcity of the data of Uruguay, it is not possible yet to extract a general conclusion. Finally, the ${ }^{40} \mathrm{~K}$ data are higher than the mean values in most of the cases, and fit into the worldwide range with some exceptions.

\begin{tabular}{|c|c|c|c|c|c|c|}
\hline Location & Code & $238 \mathrm{U}$ & $232 \mathrm{Th}$ & ${ }^{40} \mathrm{~K}$ & ${ }^{137} \mathrm{Cs}$ & Reference \\
\hline \multicolumn{7}{|c|}{ Argentina } \\
\hline $\begin{array}{l}34^{\circ} 54.45^{\prime} \mathrm{S} ; \\
58^{\circ} 8.37^{\prime} \mathrm{W}\end{array}$ & AS1 & $\mathrm{P}$ & $\mathrm{P}$ & $\mathrm{P}$ & $\mathrm{P}$ & \multirow{4}{*}{ Montes et al., 2010b } \\
\hline $\begin{array}{l}35^{\circ} 3.26^{\prime} \mathrm{S} \\
57^{\circ} 51.21^{\prime} \mathrm{W}\end{array}$ & AS2 & $\mathrm{P}$ & $\mathrm{P}$ & $\mathrm{P}$ & $\mathrm{P}$ & \\
\hline $\begin{array}{l}34^{\circ} 54.14^{\prime} \mathrm{S} ; \\
57^{\circ} 55.10^{\prime} \mathrm{W}\end{array}$ & AS3 & $\mathrm{P}$ & $\mathrm{P}$ & $\mathrm{P}$ & $\mathrm{P}$ & \\
\hline $\begin{array}{l}34^{\circ} 48.46^{\prime} \mathrm{S} ; \\
58^{\circ} 5.25^{\prime} \mathrm{W}\end{array}$ & AS4 & $\mathrm{P}$ & $\mathrm{P}$ & $\mathrm{P}$ & $\mathrm{P}$ & \\
\hline $\begin{array}{l}34^{\circ} 48.08^{\prime} \mathrm{S} ; \\
58^{\circ} 5.04^{\prime} \mathrm{W}\end{array}$ & AS5 & $S$ & $S$ & $S$ & $S$ & \multirow{4}{*}{$\begin{array}{c}\text { Valdés, M. E. et al., } 2011 \\
\text { Montes, } 2011\end{array}$} \\
\hline $\begin{array}{l}35^{\circ} 0.70^{\prime} \mathrm{S} ; \\
57^{\circ} 44.29^{\prime} \mathrm{W}\end{array}$ & AS6 & $S$ & $S$ & $S$ & $S$ & \\
\hline $\begin{array}{l}34^{\circ} 57.85 ' S \\
57^{\circ} 45.66^{\prime} \mathrm{W}\end{array}$ & AS7 & $S$ & $S$ & $S$ & $S$ & \\
\hline $\begin{array}{l}34^{\circ} 49.67^{\prime} \mathrm{S} \\
58^{\circ} 35.14 \mathrm{~W}\end{array}$ & AS8 & $S$ & $S$ & $S$ & $S$ & \\
\hline
\end{tabular}




\begin{tabular}{|c|c|c|c|c|c|c|}
\hline Location & Code & $238 U$ & ${ }^{232} \mathrm{Th}$ & ${ }^{40} K$ & ${ }^{137} \mathrm{Cs}$ & Reference \\
\hline $\begin{array}{l}34^{\circ} 49.30^{\prime} \mathrm{S} ; \\
58^{\circ} 35.14^{\prime} \mathrm{W}\end{array}$ & AS9 & S & $S$ & S & S & \\
\hline $\begin{array}{l}34^{\circ} 50.69^{\prime} \mathrm{S} ; \\
58^{\circ} 34.73^{\prime} \mathrm{W}\end{array}$ & AS10 & $S$ & $S$ & S & $S$ & \\
\hline $\begin{array}{l}34^{\circ} 50.46^{\prime} \mathrm{S} ; \\
58^{\circ} 45.13^{\prime} \mathrm{W}\end{array}$ & AS11 & $S$ & $S$ & $S$ & $S$ & \\
\hline $\begin{array}{l}33^{\circ} 50.00^{\prime} \mathrm{S} ; \\
59^{\circ} 52.00^{\prime} \mathrm{W}\end{array}$ & AS12 & & & & $\mathrm{P}$ & \multirow{4}{*}{ Bujan et al., 2000; 2003; } \\
\hline $\begin{array}{l}33^{\circ} 50.00^{\prime} \mathrm{S} ; \\
59^{\circ} 52.00^{\prime} \mathrm{W}\end{array}$ & AS13 & & & & $\mathrm{P}$ & \\
\hline $\begin{array}{l}33^{\circ} 50.00^{\prime} \mathrm{S} ; \\
59^{\circ} 52.00 \mathrm{~W}\end{array}$ & AS14 & & & & $\mathrm{P}$ & \\
\hline $\begin{array}{l}33^{\circ} 50.00^{\prime} \mathrm{S} ; \\
59^{\circ} 52.00^{\prime} \mathrm{W}\end{array}$ & AS15 & & & & $\mathrm{P}$ & \\
\hline $\begin{array}{l}33^{\circ} 40.17^{\prime} \mathrm{S} ; \\
65^{\circ} 23.45^{\prime} \mathrm{W}\end{array}$ & AS16 & & & & $\mathrm{P}$ & \multirow{7}{*}{ Jury Ayub et al., 2007} \\
\hline $\begin{array}{l}33^{\circ} 40.17^{\prime} \mathrm{S} ; \\
65^{\circ} 23.45^{\prime} \mathrm{W}\end{array}$ & AS17 & & & & $\mathrm{P}$ & \\
\hline $\begin{array}{l}33^{\circ} 40.17^{\prime} \mathrm{S} ; \\
65^{\circ} 23.45^{\prime} \mathrm{W}\end{array}$ & AS18 & & & & $\mathrm{P}$ & \\
\hline $\begin{array}{l}33^{\circ} 40.17^{\prime} \mathrm{S} ; \\
65^{\circ} 23.45^{\prime} \mathrm{W}\end{array}$ & AS19 & & & & $\mathrm{P}$ & \\
\hline $\begin{array}{l}33^{\circ} 39.93^{\prime} \mathrm{S} ; \\
65^{\circ} 23.27^{\prime} \mathrm{W}\end{array}$ & AS20 & & & & $\mathrm{P}$ & \\
\hline $\begin{array}{l}33^{\circ} 39.93^{\prime} \mathrm{S} ; \\
65^{\circ} 23.27^{\prime} \mathrm{W}\end{array}$ & AS21 & & & & $\mathrm{P}$ & \\
\hline $\begin{array}{l}3^{\circ} 39.93^{\prime} \mathrm{S} ; \\
65^{\circ} 23.27^{\prime} \mathrm{W}\end{array}$ & AS22 & & & & $\mathrm{P}$ & \\
\hline $\begin{array}{l}33^{\circ} 40.17^{\prime} \mathrm{S} ; \\
65^{\circ} 23.45^{\prime} \mathrm{W}\end{array}$ & AS23 & $\mathrm{P}$ & $\mathrm{P}$ & $\mathrm{P}$ & $\mathrm{P}$ & \multirow{2}{*}{ Jury Ayub et al., 2008} \\
\hline $\begin{array}{l}33^{\circ} 39.93^{\prime} \mathrm{S} ; \\
65^{\circ} 23.27^{\prime} \mathrm{W}\end{array}$ & AS24 & $\mathrm{P}$ & $\mathrm{P}$ & $\mathrm{P}$ & $\mathrm{P}$ & \\
\hline UNSCEAR & UN & & & $S$ & & UNSCEAR, 2008 \\
\hline \multicolumn{7}{|c|}{ Brazil } \\
\hline $\begin{array}{l}\text { Rio } \\
\text { Grande do } \\
\text { Norte }\end{array}$ & BS1 & S & $S$ & S & & Malanca et al, 1996 \\
\hline $\begin{array}{l}22^{\circ} 42.00^{\prime} \mathrm{S} ; \\
47^{\circ} 38.00^{\prime} \mathrm{W}\end{array}$ & BS2 & & & & $\mathrm{P}$ & \multirow{4}{*}{ Corrochel et al, 2005} \\
\hline $\begin{array}{l}22^{\circ} 47.00^{\prime} \mathrm{S} ; \\
47^{\circ} 19.00^{\prime} \mathrm{W}\end{array}$ & BS3 & & & & $\mathrm{P}$ & \\
\hline $\begin{array}{l}22^{\circ} 09.00^{\prime} \mathrm{S} ; \\
47^{\circ} 01.00^{\prime} \mathrm{W}\end{array}$ & BS4 & & & & $\mathrm{P}$ & \\
\hline $\begin{array}{l}22^{\circ} 40.00^{\prime} \mathrm{S} ; \\
48^{\circ} 10.00^{\prime} \mathrm{W}\end{array}$ & BS5 & & & & $\mathrm{P}$ & \\
\hline
\end{tabular}




\begin{tabular}{|c|c|c|c|c|c|c|}
\hline Location & Code & $238 \mathrm{U}$ & $232 \mathrm{Th}$ & ${ }^{40} \mathrm{~K}$ & ${ }^{137} \mathrm{Cs}$ & Reference \\
\hline $\begin{array}{l}01^{\circ} 57.00^{\prime} \mathrm{S} ; \\
54^{\circ} 12.00^{\prime} \mathrm{W}\end{array}$ & BS6 & & & & $\mathrm{P}$ & \multirow{21}{*}{$\begin{array}{l}\text { Handl et al, } 2008 \\
\text { Handl et al, } 2008\end{array}$} \\
\hline $\begin{array}{l}03^{\circ} 08.00^{\prime} \mathrm{S} ; \\
60^{\circ} 01.00^{\prime} \mathrm{W}\end{array}$ & BS7 & & & & $\mathrm{P}$ & \\
\hline $\begin{array}{l}03^{\circ} 08.00^{\prime} \mathrm{S} ; \\
60^{\circ} 01.00^{\prime} \mathrm{W}\end{array}$ & BS8 & & & & $\mathrm{P}$ & \\
\hline $\begin{array}{l}08^{\circ} 10.00^{\prime} \mathrm{S} ; \\
34^{\circ} 54.00^{\prime} \mathrm{W}\end{array}$ & BS9 & & & & $\mathrm{P}$ & \\
\hline $\begin{array}{l}09^{\circ} 26.00^{\prime} \mathrm{S} ; \\
38^{\circ} 08.00^{\prime} \mathrm{W}\end{array}$ & BS10 & & & & $\mathrm{P}$ & \\
\hline $\begin{array}{l}09^{\circ} 26.00^{\prime} \mathrm{S} ; \\
38^{\circ} 08.00^{\prime} \mathrm{W}\end{array}$ & BS11 & & & & $\mathrm{P}$ & \\
\hline $\begin{array}{l}15^{\circ} 58.00^{\prime} \mathrm{S} ; \\
47^{\circ} 59.00^{\prime} \mathrm{W}\end{array}$ & BS12 & & & & $\mathrm{P}$ & \\
\hline $\begin{array}{l}16^{\circ} 42.00^{\prime} \mathrm{S} ; \\
47^{\circ} 40.00^{\prime} \mathrm{W}\end{array}$ & BS13 & & & & $\mathrm{P}$ & \\
\hline $\begin{array}{l}19^{\circ} 29.00^{\prime} \mathrm{S} ; \\
57^{\circ} 25.00^{\prime} \mathrm{W}\end{array}$ & BS14 & & & & $\mathrm{P}$ & \\
\hline $\begin{array}{l}20^{\circ} 43.00^{\prime} \mathrm{S} ; \\
54^{\circ} 31.00^{\prime} \mathrm{W}\end{array}$ & BS15 & & & & $\mathrm{P}$ & \\
\hline $\begin{array}{l}20^{\circ} 22.00^{\prime} \mathrm{S} ; \\
43^{\circ} 24.00^{\prime} \mathrm{W}\end{array}$ & BS16 & & & & $\mathrm{P}$ & \\
\hline $\begin{array}{l}20^{\circ} 21.00^{\prime} \mathrm{S} ; \\
43^{\circ} 29.00^{\prime} \mathrm{W}\end{array}$ & BS17 & & & & $\mathrm{P}$ & \\
\hline $\begin{array}{l}22^{\circ} 20.00^{\prime} \mathrm{S} ; \\
43^{\circ} 37.00^{\prime} \mathrm{W}\end{array}$ & BS18 & & & & $\mathrm{P}$ & \\
\hline $\begin{array}{l}22^{\circ} 30.00^{\prime} \mathrm{S} ; \\
44^{\circ} 30.00^{\prime} \mathrm{W}\end{array}$ & BS19 & & & & $\mathrm{P}$ & \\
\hline $\begin{array}{l}22^{\circ} 20.00^{\prime} \mathrm{S} ; \\
44^{\circ} 40.00^{\prime} \mathrm{W}\end{array}$ & BS20 & & & & $\mathrm{P}$ & \\
\hline $\begin{array}{l}23^{\circ} 10.00^{\prime} \mathrm{S} ; \\
44^{\circ} 11.00^{\prime} \mathrm{W}\end{array}$ & BS21 & & & & $\mathrm{P}$ & \\
\hline $\begin{array}{l}23^{\circ} 07.00^{\prime} \mathrm{S} ; \\
44^{\circ} 10.00^{\prime} \mathrm{W}\end{array}$ & BS22 & & & & $\mathrm{P}$ & \\
\hline $\begin{array}{l}25^{\circ} 17.00^{\prime} \mathrm{S} ; \\
48^{\circ} 55.00^{\prime} \mathrm{W}\end{array}$ & BS23 & & & & $\mathrm{P}$ & \\
\hline $\begin{array}{l}26^{\circ} 39.00^{\prime} \mathrm{S} ; \\
48^{\circ} 41.00^{\prime} \mathrm{W}\end{array}$ & BS24 & & & & $\mathrm{P}$ & \\
\hline $\begin{array}{l}29^{\circ} 21.00^{\prime} \mathrm{S} ; \\
50^{\circ} 51.00^{\prime} \mathrm{W}\end{array}$ & BS25 & & & & $\mathrm{P}$ & \\
\hline $\begin{array}{l}30^{\circ} 05.00^{\prime} \mathrm{S} ; \\
51^{\circ} 36.00^{\prime} \mathrm{W}\end{array}$ & BS26 & & & & $\mathrm{P}$ & \\
\hline
\end{tabular}




\begin{tabular}{|c|c|c|c|c|c|c|}
\hline Location & Code & ${ }^{238} U$ & $232 \mathrm{Th}$ & ${ }^{40} \mathrm{~K}$ & ${ }^{137} \mathrm{Cs}$ & Reference \\
\hline \multicolumn{7}{|c|}{ Uruguay } \\
\hline $\begin{array}{l}32^{\circ} 26.00^{\prime} \mathrm{S} ; \\
54^{\circ} 19.00^{\prime} \mathrm{W}\end{array}$ & US1 & S & & & S & \multirow{8}{*}{ Odino Moure, 2010} \\
\hline $\begin{array}{l}31^{\circ} 18.00^{\prime} \mathrm{S} ; \\
57^{\circ} 02.00^{\prime} \mathrm{W}\end{array}$ & US2 & S & & & S & \\
\hline $\begin{array}{l}34^{\circ} 20.00^{\prime} \mathrm{S} ; \\
56^{\circ} 43.00^{\prime} \mathrm{W}\end{array}$ & US3 & S & & & $S$ & \\
\hline $\begin{array}{l}34^{\circ} 10.00^{\prime} \mathrm{S} ; \\
57^{\circ} 41.00^{\prime} \mathrm{W} \\
(2004)\end{array}$ & US4 & $S$ & & & $S$ & \\
\hline $\begin{array}{l}34^{\circ} 10.00^{\prime} \mathrm{S} ; \\
57^{\circ} 41.00^{\prime} \mathrm{W} \\
(2005)\end{array}$ & US5 & S & & & S & \\
\hline $\begin{array}{l}34^{\circ} 10.00^{\prime} \mathrm{S} ; \\
57^{\circ} 41.00^{\prime} \mathrm{W} \\
(2006)\end{array}$ & US6 & $S$ & & & $S$ & \\
\hline $\begin{array}{l}34^{\circ} 10.00^{\prime} \mathrm{S} ; \\
57^{\circ} 41.00^{\prime} \mathrm{W} \\
(2007)\end{array}$ & US7 & S & & & S & \\
\hline $\begin{array}{l}34^{\circ} 10.00^{\prime} \mathrm{S} ; \\
57^{\circ} 41.00^{\prime} \mathrm{W} \\
(2009)\end{array}$ & US8 & S & & & S & \\
\hline \multicolumn{7}{|c|}{ Venezuela } \\
\hline Guaña & VS1 & & & & $S$ & Sajó-Bohus et al, 1999 \\
\hline \multicolumn{7}{|c|}{ Chile } \\
\hline $\begin{array}{l}39^{\circ} 44.00^{\prime} \mathrm{S} ; \\
73^{\circ} 22.80^{\prime} \mathrm{W}\end{array}$ & CS1 & & & & $\mathrm{P}$ & \multirow{4}{*}{ Schuller et al., 1997} \\
\hline $\begin{array}{l}38^{\circ} 41.50^{\prime} \mathrm{S} ; \\
72^{\circ} 53.00^{\prime} \mathrm{W}\end{array}$ & CS2 & & & & $\mathrm{P}$ & \\
\hline $\begin{array}{l}39^{\circ} 41.30^{\prime} \mathrm{S} ; \\
72^{\circ} 57.10^{\prime} \mathrm{W}\end{array}$ & CS3 & & & & $\mathrm{P}$ & \\
\hline $\begin{array}{l}40^{\circ} 23.00^{\prime} \mathrm{S} ; \\
72^{\circ} 57.50^{\prime} \mathrm{W}\end{array}$ & CS4 & & & & $\mathrm{P}$ & \\
\hline 1 & CS5 & & & & $\mathrm{P}$ & \multirow{13}{*}{ Schuller et al., 2002} \\
\hline 2 & CS6 & & & & $\mathrm{P}$ & \\
\hline 3 & CS7 & & & & $\mathrm{P}$ & \\
\hline 4 & CS8 & & & & $\mathrm{P}$ & \\
\hline 5 & CS9 & & & & $\mathrm{P}$ & \\
\hline 6 & CS10 & & & & $\mathrm{P}$ & \\
\hline 7 & CS11 & & & & $\mathrm{P}$ & \\
\hline 8 & CS12 & & & & $\mathrm{P}$ & \\
\hline 9 & CS13 & & & & $\mathrm{P}$ & \\
\hline 10 & CS14 & & & & $\mathrm{P}$ & \\
\hline 11 & CS15 & & & & $\mathrm{P}$ & \\
\hline 12 & CS16 & & & & $\mathrm{P}$ & \\
\hline 13 & CS17 & & & & $\mathrm{P}$ & \\
\hline
\end{tabular}




\begin{tabular}{|c|c|c|c|c|c|c|}
\hline Location & Code & $238 U$ & $232 \mathrm{Th}$ & ${ }^{40} \mathrm{~K}$ & ${ }^{137} \mathrm{Cs}$ & Reference \\
\hline 14 & CS18 & & & & $\mathrm{P}$ & \\
\hline 15 & CS19 & & & & $\mathrm{P}$ & \\
\hline 16 & CS20 & & & & $\mathrm{P}$ & \\
\hline 17 & CS21 & & & & $\mathrm{P}$ & \\
\hline 18 & CS22 & & & & $\mathrm{P}$ & \\
\hline 19 & CS23 & & & & $\mathrm{P}$ & \\
\hline 20 & CS24 & & & & $\mathrm{P}$ & \\
\hline 21 & CS25 & & & & $\bar{P}$ & \\
\hline 22 & CS26 & & & & $\mathrm{P}$ & \\
\hline 23 & CS27 & & & & $\mathrm{P}$ & \\
\hline 24 & CS28 & & & & $\mathrm{P}$ & \\
\hline 25 & CS29 & & & & $\mathrm{P}$ & \\
\hline 26 & CS30 & & & & $\mathrm{P}$ & \\
\hline 27 & CS31 & & & & $\mathrm{P}$ & \\
\hline 28 & CS32 & & & & $\mathrm{P}$ & \\
\hline 29 & CS33 & & & & $\mathrm{P}$ & \\
\hline $\begin{array}{l}50^{\circ} 53.00^{\prime} \mathrm{S} ; \\
72^{\circ} 40.00^{\prime} \mathrm{W}\end{array}$ & CS34 & & & & $\mathrm{P}$ & \multirow{14}{*}{ Schuller et al., 2004} \\
\hline $\begin{array}{l}51^{\circ} 08.00^{\prime} \mathrm{S} ; \\
53^{\circ} 10.00^{\prime} \mathrm{W}\end{array}$ & CS35 & & & & P & \\
\hline $\begin{array}{l}51^{\circ} 10.00^{\prime} \mathrm{S} ; \\
73^{\circ} 05.00^{\prime} \mathrm{W}\end{array}$ & CS36 & & & & $\mathrm{P}$ & \\
\hline $\begin{array}{l}51^{\circ} 12.00^{\prime} \mathrm{S} ; \\
73^{\circ} 00.00^{\prime} \mathrm{W}\end{array}$ & CS37 & & & & P & \\
\hline $\begin{array}{l}52^{\circ} 20.00^{\prime} \mathrm{S} ; \\
68^{\circ} 25.00^{\prime} \mathrm{W}\end{array}$ & CS38 & & & & $\mathrm{P}$ & \\
\hline $\begin{array}{l}52^{\circ} 16.00^{\prime} \mathrm{S} ; \\
68^{\circ} 50.00^{\prime} \mathrm{W}\end{array}$ & CS39 & & & & P & \\
\hline $\begin{array}{l}52^{\circ} 35.00^{\prime} \mathrm{S} ; \\
69^{\circ} 50.00^{\prime} \mathrm{W}\end{array}$ & CS40 & & & & $\mathrm{P}$ & \\
\hline $\begin{array}{l}52^{\circ} 38.00^{\prime} \mathrm{S} ; \\
70^{\circ} 15.00^{\prime} \mathrm{W}\end{array}$ & CS41 & & & & P & \\
\hline $\begin{array}{l}52^{\circ} 40.00^{\prime} \mathrm{S} ; \\
70^{\circ} 50.00^{\prime} \mathrm{W}\end{array}$ & CS42 & & & & P & \\
\hline $\begin{array}{l}52^{\circ} 25.00^{\prime} \mathrm{S} ; \\
71^{\circ} 25.00^{\prime} \mathrm{W}\end{array}$ & CS43 & & & & P & \\
\hline $\begin{array}{l}52^{\circ} 35.00^{\prime} \mathrm{S} ; \\
71^{\circ} 33.00^{\prime} \mathrm{W}\end{array}$ & CS44 & & & & $\mathrm{P}$ & \\
\hline $\begin{array}{l}51^{\circ} 55.00^{\prime} \mathrm{S} ; \\
72^{\circ} 00.00^{\prime} \mathrm{W}\end{array}$ & CS45 & & & & P & \\
\hline $\begin{array}{l}52^{\circ} 35.00^{\prime} \mathrm{S} ; \\
71^{\circ} 42.00^{\prime} \mathrm{W}\end{array}$ & CS46 & & & & $\mathrm{P}$ & \\
\hline $\begin{array}{l}53^{\circ} 36.00^{\prime} \mathrm{S} ; \\
70^{\circ} 50.00^{\prime} \mathrm{W}\end{array}$ & CS47 & & & & P & \\
\hline
\end{tabular}

Table 1. Location, type of survey and monitored nuclides in soils of South America. S: surface activity determinations and P: profile activity determination. 


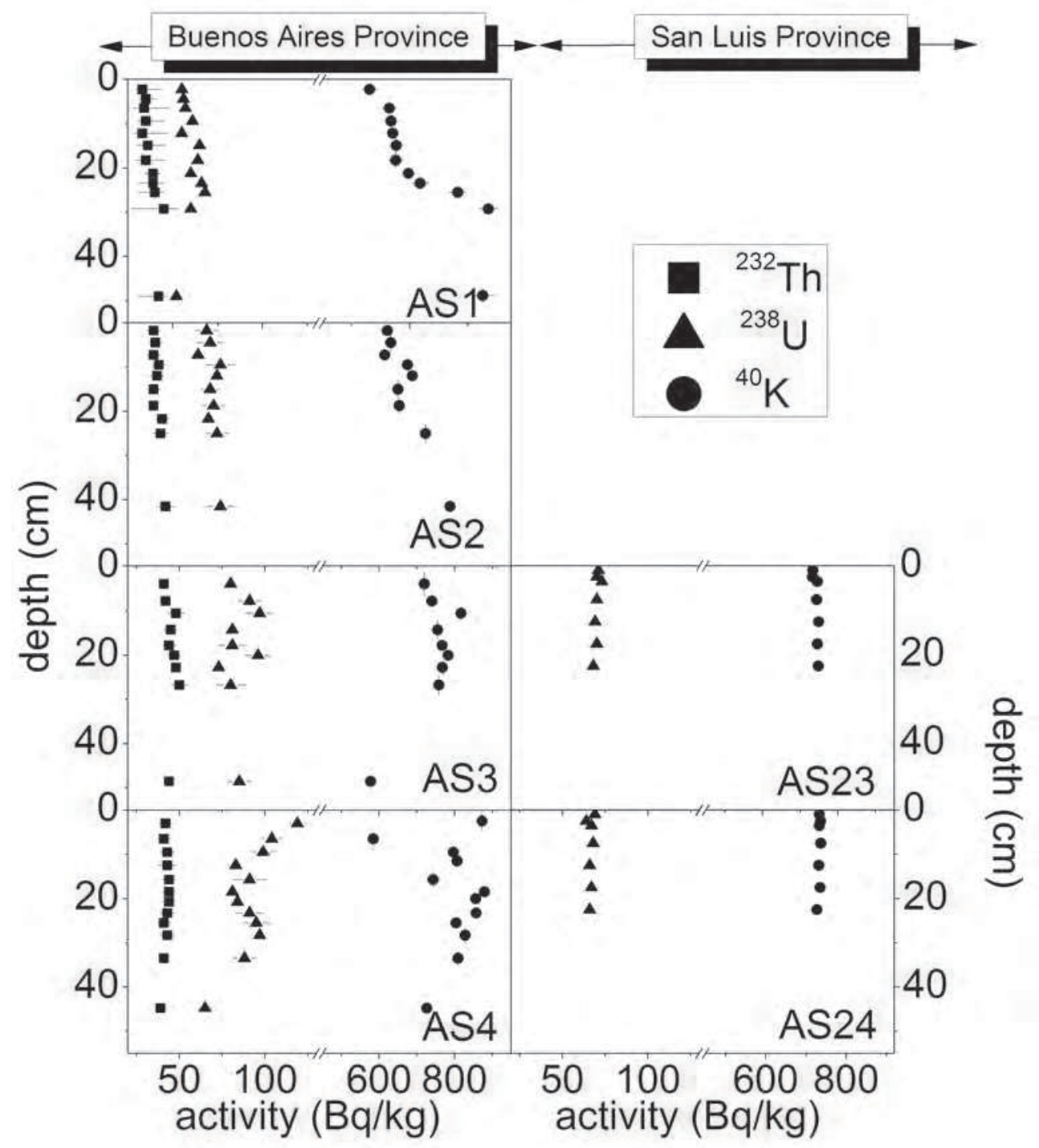

Fig. 2. Depth distribution activity of ${ }^{232} \mathrm{The},{ }^{238} \mathrm{U}$ and ${ }^{40} \mathrm{~K}$ in Argentina, labelled with the sample code. 


\begin{tabular}{|c|c|c|c|c|c|c|c|}
\hline code & $\begin{array}{c}{ }^{238} \mathrm{U} \\
(\mathrm{Bq} / \mathrm{kg})\end{array}$ & $\begin{array}{c}232 \mathrm{Th} \\
(\mathrm{Bq} / \mathrm{kg})\end{array}$ & $\begin{array}{c}{ }^{40} \mathrm{~K} \\
(\mathrm{~Bq} / \mathrm{kg})\end{array}$ & Code & $\begin{array}{c}238 \mathrm{U} \\
(\mathrm{Bq} / \mathrm{kg})\end{array}$ & $\begin{array}{c}232 \mathrm{Th} \\
(\mathrm{Bq} / \mathrm{kg})\end{array}$ & $\begin{array}{c}{ }^{40} \mathrm{~K} \\
(\mathrm{~Bq} / \mathrm{kg})\end{array}$ \\
\hline AS1 & $55 \pm 6$ & $33 \pm 4$ & $531 \pm 13$ & BS1 & $10-136.7$ & $12-191$ & 56-1972 \\
\hline AS2 & $66 \pm 7$ & $35 \pm 4$ & $622 \pm 15$ & US1 & $19 \pm 2$ & $75 \pm 7$ & $1054 \pm 100$ \\
\hline AS3 & $80 \pm 4$ & $41 \pm 2$ & $720 \pm 14$ & US2 & $7.2 \pm 0.5$ & $11 \pm 1$ & $90 \pm 5$ \\
\hline AS4 & $119 \pm 5$ & $42 \pm 4$ & $717 \pm 15$ & US3 & $23 \pm 2$ & $51 \pm 5$ & $440 \pm 40$ \\
\hline AS5 & $106 \pm 10$ & $43 \pm 4$ & $873 \pm 18$ & US4 & $19 \pm 2$ & $8.6 \pm 0.5$ & $492 \pm 45$ \\
\hline AS6 & $61 \pm 8$ & $35 \pm 2$ & $576 \pm 15$ & US5 & $22 \pm 2$ & $36 \pm 31$ & $560 \pm 51$ \\
\hline AS7 & $52 \pm 9$ & $30 \pm 4$ & $658 \pm 17$ & US6 & $21 \pm 2$ & $35 \pm 30$ & $495 \pm 45$ \\
\hline AS8 & $65 \pm 18$ & $35 \pm 17$ & $644 \pm 29$ & US7 & $7.7 \pm 0.5$ & $9.4 \pm 0.5$ & $255 \pm 21$ \\
\hline AS9 & $57 \pm 11$ & $27 \pm 16$ & $498 \pm 25$ & US8 & $14 \pm 1$ & $19 \pm 2$ & $340 \pm 31$ \\
\hline AS10 & $53 \pm 13$ & $24 \pm 12$ & $470 \pm 22$ & WA & 35 & 30 & 400 \\
\hline AS11 & $52 \pm 10$ & $32 \pm 7$ & $547 \pm 23$ & WR & $16-110$ & $11-64$ & $140-850$ \\
\hline AS23 & $71 \pm 4$ & - & $733 \pm 11$ & & & & \\
\hline AS24 & $69 \pm 4$ & - & $734 \pm 20$ & & & & \\
\hline $\mathrm{UN}$ & - & - & $540-750$ & & & & \\
\hline
\end{tabular}

Table 2. Natural surface activity concentrations in soils. WA: worldwide average and WR: worldwide range [UNSCEAR, 2000; UNSCEAR, 2008]. 




Fig. 3. Natural activity of ${ }^{40} \mathrm{~K},{ }^{232} \mathrm{Th}$ and ${ }^{238} \mathrm{U}$ in soil surface. The dash line corresponds to the UNSCEAR average worldwide values [UNSCEAR, 2000]. The vertical bars correspond to the experimental errors in the case of Argentina and Uruguay, and to the standard deviation of a set of determinations in the case of Brazil.

\subsection{Annual committed effective dose by external irradiation calculations}

The contribution of natural nuclides to the absorbed dose rate at $1 \mathrm{~m}$ above the ground depends on the concentration of radionuclides in the soil. There is a direct relationship between terrestrial gamma radiation dose and radionuclide natural concentrations in soils. The exposure dose rate can be evaluated accounting for the activity values of the nuclides $\left(A_{i}\right)$ and the conversion factors $\left(f_{i}\right)$. These coefficients are reported in Table 3 [UNSCEAR, 2000; UNSCEAR, 2008]. Based in the analysis of the UNSCEAR 1982 report [UNSCEAR, 1982], the International Committee of Radiation Protection (ICRP) used a coefficient $\left(C_{i}\right)$ to 
convert the absorbed dose in air to annual committed effective dose (aced). Monte Carlo Calculations radiation- transport codes indicate that higher values should be used for infant and children. These values are quoted in the Table 3 . To calculate the annual effective dose it has also considered that the spent time outdoors is $20 \%$ of total time [USNCEAR, 2008], i.e.:

$$
\text { aced }(\mathrm{Sv})=10^{-9} \times 24 \times 365 \times \mathrm{C}_{\mathrm{c}} \times 0.2 \times \sum_{\mathrm{i}} \mathrm{f}_{\mathrm{i}} \mathrm{A}_{\mathrm{i}} \mathrm{C}_{\mathrm{i}}
$$

\begin{tabular}{|c|c|c|c|c|}
\hline nuclide & $f_{i}\left(n \mathrm{Gyh}^{-1} / \mathrm{B}_{\mathrm{k} k g^{-1}}\right)$ & \multicolumn{3}{|c|}{$C_{i}(\mathrm{~Sv} / \mathrm{Gy})$} \\
\hline & & infants & children & adults \\
\hline${ }^{40} \mathrm{~K}$ & 0.0417 & 0.926 & 0.803 & 0.709 \\
\hline${ }^{232} \mathrm{Th}$ & 0.604 & 0.907 & 0.798 & 0.695 \\
\hline${ }^{238 \mathrm{U}}$ & 0.462 & 0.899 & 0.766 & 0.672 \\
\hline Average & & 0.91 & 0.79 & 0.69 \\
\hline
\end{tabular}

Table 3. Conversion factors $\left(f_{i}\right)$ and absorbed dose to effective dose equivalent conversion coefficients $\left(C_{i}\right)$ [UNSCEAR 2008, UNSCEAR 2000].

\begin{tabular}{|c|c|c|c|c|c|c|c|}
\hline & \multicolumn{3}{|c|}{ aced $(m S v)$} & \multicolumn{4}{|c|}{ aced $(m S v)$} \\
\hline code & infants & children & adults & code & infants & children & adults \\
\hline AS1 & $0.108 \pm 0.006$ & $0.093 \pm 0.005$ & $0.082 \pm 0.005$ & BS1 & $0.03-0.42$ & $0.02-0.36$ & $0.02-0.32$ \\
\hline AS2 & $0.134 \pm 0.007$ & $0.107 \pm 0.006$ & $0.094 \pm 0.005$ & US1 & $0.16 \pm 0.01$ & $0.137 \pm 0.009$ & $0.120 \pm 0.008$ \\
\hline AS3 & $0.146 \pm 0.004$ & $0.126 \pm 0.003$ & $0.111 \pm 0.003$ & US2 & $0.022 \pm 0.001$ & $0.019 \pm 0.001$ & $0.0667 \pm 0.0009$ \\
\hline AS4 & $0.175 \pm 0.006$ & $0.151 \pm 0.005$ & $0.133 \pm 0.004$ & US3 & $0.095 \pm 0.006$ & $0.083 \pm 0.005$ & $0.073 \pm 0.005$ \\
\hline AS5 & $0.177 \pm 0.009$ & $0.153 \pm 0.007$ & $0.135 \pm 0.007$ & US4 & $0.055 \pm 0.004$ & $0.048 \pm 0.003$ & $0.042 \pm 0.003$ \\
\hline AS6 & $0.117 \pm 0.007$ & $0.101 \pm 0.006$ & $0.089 \pm 0.005$ & US5 & $0.09 \pm 0.03$ & $0.08 \pm 0.03$ & $0.07 \pm 0.03$ \\
\hline AS7 & $0.111 \pm 0.008$ & $0.096 \pm 0.007$ & $0.084 \pm 0.006$ & US6 & $0.08 \pm 0.03$ & $0.07 \pm 0.03$ & $0.06 \pm 0.02$ \\
\hline AS8 & $0.12 \pm 0.02$ & $0.11 \pm 0.02$ & $0.09 \pm 0.02$ & US7 & $0.032 \pm 0.002$ & $0.028 \pm 0.002$ & $0.024 \pm 0.001$ \\
\hline AS9 & $0.10 \pm 0.02$ & $0.09 \pm 0.02$ & $0.08 \pm 0.02$ & US8 & $0.051 \pm 0.003$ & $0.0447 \pm 0.003$ & $0.039 \pm 0.003$ \\
\hline AS10 & $0.09 \pm 0.02$ & $0.08 \pm 0.01$ & $0.07 \pm 0.01$ & & & & \\
\hline AS11 & $0.11 \pm 0.01$ & $0.091 \pm 0.009$ & $0.080 \pm 0.008$ & & & & \\
\hline
\end{tabular}

Table 4. Calculated annual committed effective terrestrial exposure dose for infants, children and adults.

It is worth to mention that in the case of adults, the calculated annual committed effective doses due to terrestrial external exposure resulted slightly higher than the UNSCEAR reported values [UNSCEAR, 2000], as observed in Fig. 4. 


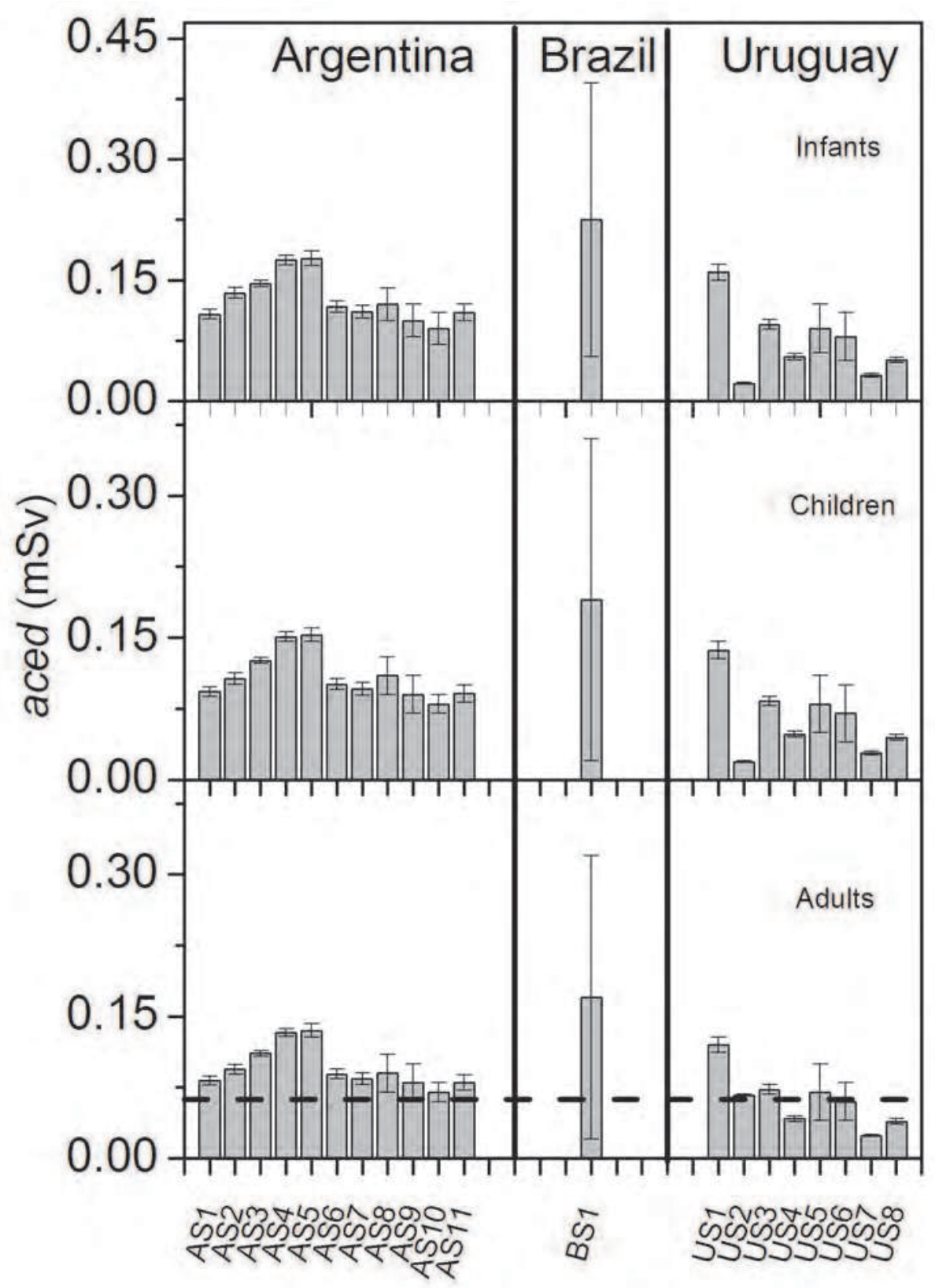

Fig. 4. Calculated annual committed effective dose for infants, children and adults. The dash line corresponds to the UNSCEAR reported values [UNSCEAR, 2000]. The vertical bars correspond to the experimental errors in the case of Argentina and Uruguay, and to the standard deviation of a set of determinations in the case of Brazil. 


\subsection{Anthropogenic nuclides}

In the last decades, ${ }^{137} \mathrm{Cs}$ was the only monitored anthropogenic nuclide (gamma emitter) in the Southern Hemisphere. Aimed in the study of soil erosion, ${ }^{137} \mathrm{Cs}$ reference activity profiles (Fig. 5) were determined in the Pampa Ondulada of the Buenos Aires Province region, Argentina (AS12-AS15) [Bujan et al., 2000; Bujan et al., 2003]. The ${ }^{137} \mathrm{Cs}$ activities determined down to $90 \mathrm{~cm}$ declined sharply from the surface to the first $20 \mathrm{~cm}$, the maxima activity was observed at the top layer. An average value of $1108 \mathrm{~Bq} / \mathrm{m}^{2}$ was obtained for the local inventory. In the La Plata city region, in spite that the ${ }^{137} \mathrm{Cs}$ integrated activities of the profiles obtained down to $50 \mathrm{~cm}$ were similar in all soils, differences in the ${ }^{137} \mathrm{Cs}$ depth distributions were detected (Fig. 5). The profiles of AS1 and AS3 sites followed a Gaussian-type feature, typical of a convective-diffusive process [Likar et al., 2001; Bossen \& Kirchner, 2004]. The profile of AS2 was quite different since a Gaussian-shape was established down to $7 \mathrm{~cm}$ in depth. In the case of the AS4 soil, placed at $5 \mathrm{~km}$ from the La Plata river coast, the activity values were high at the surface and then suddenly decreased. Both facts, the high values at the surface and the deviation from the Gaussian shape [Likar et al., 2001;Bossen \& Kirchner, 2004], could be explained considering the fine texture and the flat relief of the region which induce water-logging, i.e., this area shows a low permeability of the underlying horizons, and the phreatic water affects the deepest horizons [Imbellone, 2009]. It has been claimed that Cs is sorbed by $\mathrm{Fe}_{3} \mathrm{O}_{4}$ [Singh et al., 2009; Catallette et al., 1998; Marnier \& Fromage, 2000]. However, by comparing the ${ }^{137} \mathrm{Cs}$ profiles and the Mössbauer relative fraction of $\mathrm{Fe}_{3} \mathrm{O}_{4}$ as well as with the other iron species [Montes et al.; 2010b], it was not observed an apparent correlation. A series of surface studies were also performed in the Buenos Aires Province in the neighbourhood of the Centro Atómico Ezeiza (AS5-AS11) showing that the activity concentration values down to $10 \mathrm{~cm}$ ranged between $0.9 \mathrm{~Bq} / \mathrm{kg}$ and $2.6 \mathrm{~Bq} / \mathrm{kg}$ [Vadés et al., 2011]. These values are consistent with the top layer activity data obtained from the profiles AS1-AS4 [Montes et al.; 2010b]. Vertical migration of ${ }^{137} \mathrm{Cs}$ was studied in soils of natural and semi-natural grassland areas of San Luis Province (AS16- AS21, AS23 and AS24) [Juri Ayub et al., 2007; Juri Ayub et al., 2008]. The inventories ranged from 330 $\mathrm{Bq} / \mathrm{m}^{2}$ to $730 \mathrm{~Bq} / \mathrm{m}^{2}$, while depth profiles had different shapes (see Fig. 5).

As observed in Fig. 6, differences in the patterns of ${ }^{137} \mathrm{Cs}$ depth distribution in the soil profiles of the different regions were found in the four studied sites of the South-Central region of Brazil (BS2-BS5), ascribed to chemical, physical, mineralogical and biological differences of the soils [Correchel et al., 2005]. The variability of the soil characteristics was not able to explain the spatial variability of the profiles. The average inventories of the four studied sites were $268 \mathrm{~Bq} / \mathrm{m}^{2}$, and the maximum activity value was detected at the top layer. The spatial distribution and behaviour of the ${ }^{137} \mathrm{Cs}$ in tropical, subtropical and equatorial unperturbed Brazilian soils have been investigated up to $40 \mathrm{~cm}$ (BS6-BS26) [Handl et al., 2008]. The shape of all 23 sampled sites depth profiles varied between the two ones showed in Fig. 6 . The majority of the Cs content was observed in the $10-15 \mathrm{~cm}$ top layer while minor quantities were detected down to $35 \mathrm{~cm}$. Low deposition densities were observed at the Amazon region where ascendant convection of water vapour is intense, while the south area exhibited considerable large concentrations. No correlation was observed between altitude and ${ }^{137} \mathrm{Cs}$ concentration. On the contrary, the results were correlated with the climatic de Martonne index, suggesting that the process can not be explained with single meteorological parameters [Handl et al., 2008]. 


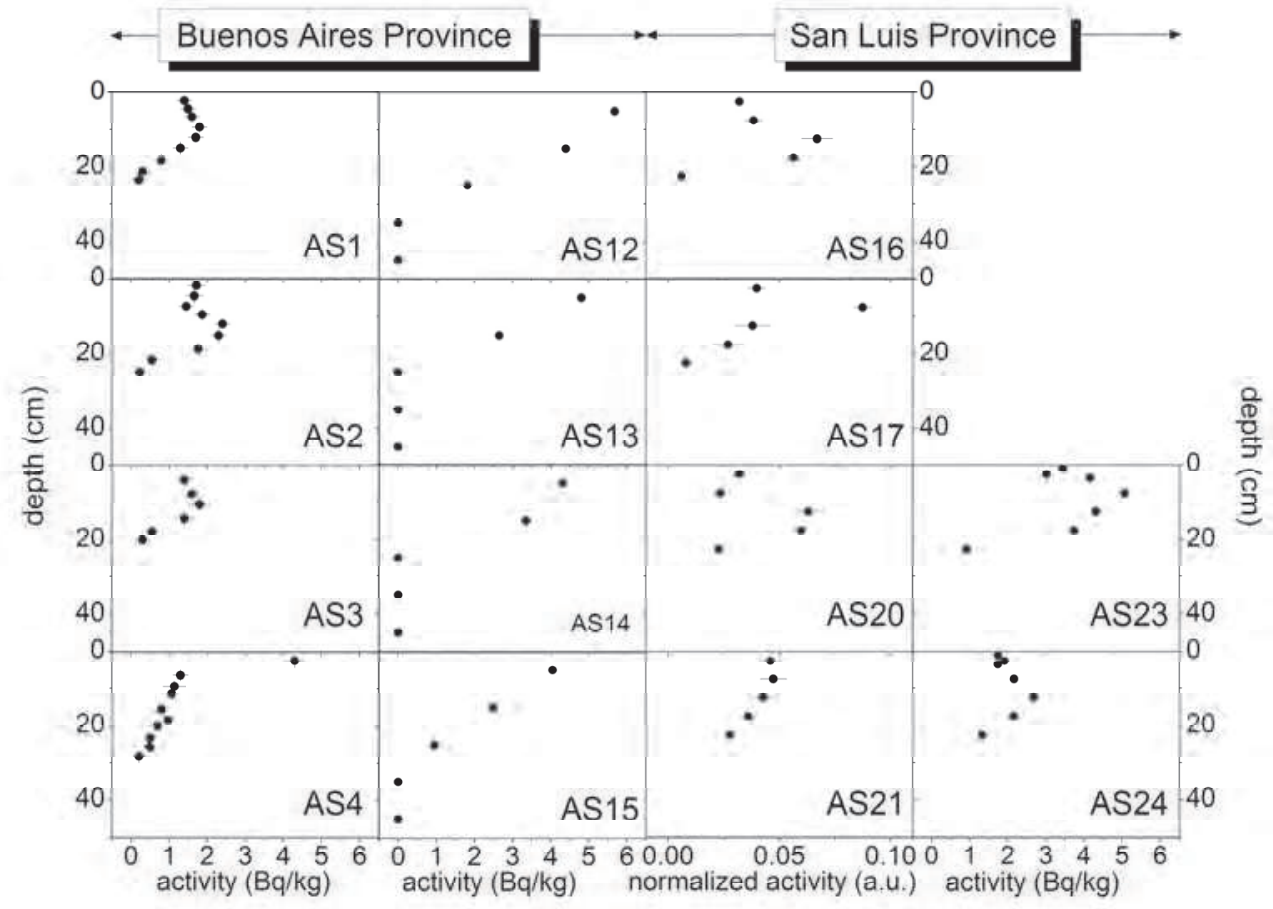

Fig. $5 .{ }^{137} \mathrm{Cs}$ depth profiles recorded in Argentina, labelled with the site code.

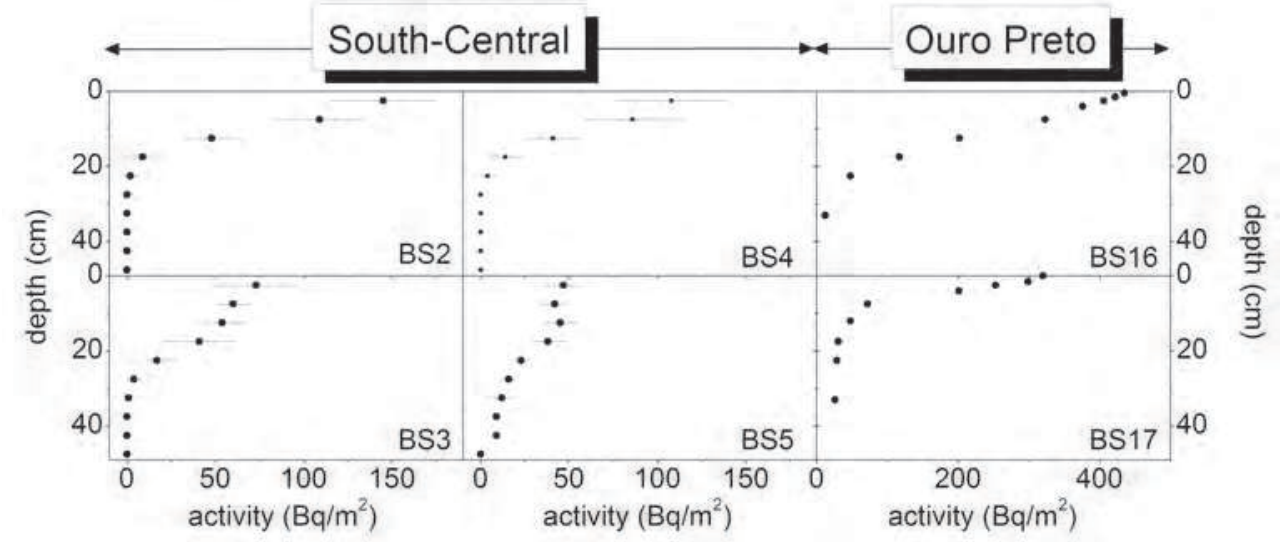

Fig. 6. ${ }^{137}$ Cs depth profiles recorded in Brazil, labelled with the site code. 
In Chile, total inventories and depth distributions of ${ }^{137} \mathrm{Cs}$ (Fig. 7) were determined at four sites of two agriculturally used soil types (CS1-CS4). The inventories were always higher than previously estimated for the Southern Hemisphere and depend on annual rainfall [Schuller et al, (1997)]. The depth distribution of ${ }^{137} \mathrm{Cs}$ in well-developed agricultural soil at 28 sites in different southern regions (CS5-CS33) was also studied [Schuller et al, 2002]. The profiles in most of the sites followed no systematic pattern in the upper few centimetres (Fig. 7), but below this depth an exponential behaviour was observed. The calculated relaxation depth [Schuller et al, 2002] ranged from $4.4 \mathrm{~cm}$ in Palehumults to $8.4 \mathrm{~cm}$ and 9.7 $\mathrm{cm}$ in Hapludands and Psamments soil types, respectively. The relaxation depth increased with decreasing clay content and increasing volume of coarse pores. Activity densities ranged from $450 \mathrm{~Bq} / \mathrm{m}^{2}$ to $5410 \mathrm{~Bq} / \mathrm{m}^{2}$, correlating with the mean annual rainfall rate of the sampling sites. The South Patagonia sheep-farming region (CS34-CS47) was studied (Fig. 7). The areal activity density varied from $222 \mathrm{~Bq} / \mathrm{m}^{2}$ to $858 \mathrm{~Bq} / \mathrm{m}^{2}$, positively correlated with the mean annual precipitation rate [Schuller et al., 2004].

In Venezuela, the ${ }^{137} \mathrm{Cs}$ concentration at two different depths, $0 \mathrm{~cm}-20 \mathrm{~cm}$ and $20 \mathrm{~cm}-40$ $\mathrm{cm}$, (VS1) was measured being the activity concentration around $0.5 \mathrm{~Bq} / \mathrm{kg}$ and $10 \mathrm{~Bq} / \mathrm{kg}$ at $20 \mathrm{~cm}$ in depth for the littoral and central regions, respectively [Sajó-Bohus et al., 1999].

Finally, the obtained values of ${ }^{137} \mathrm{Cs}$ surface activity concentration in different Uruguayan departments varied from $1.2 \mathrm{~Bq} / \mathrm{kg}$ to $2.3 \mathrm{~Bq} / \mathrm{kg}$ in the 2004 to 2009 period (US1-US8) [Odino Moure, 2010]. A spatial variation was also observed.

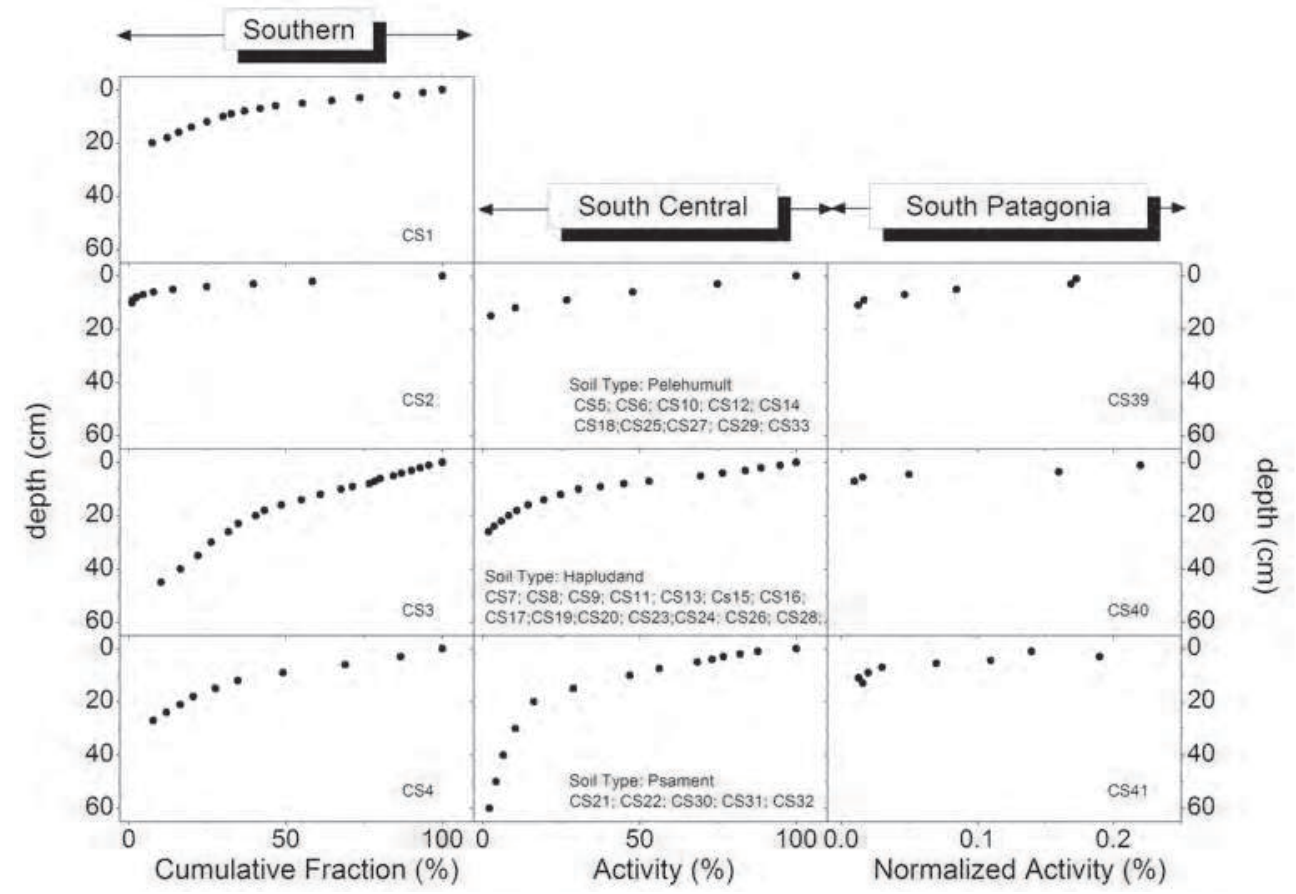

Fig. 7. ${ }^{137}$ Cs depth profiles recorded in Chile, labelled with the site code. 


\subsection{Cs inventories analysis}

The cumulated annual deposition of ${ }^{90} \mathrm{Sr}$ was compiled by UNSCEAR [UNSCEAR 2000; UNSCEAR 2008]. It is worth to mention that data of ${ }^{137} \mathrm{Cs}$ are not available due to the technological limitations on the detection of gamma emitters of the survey period. However, there is experimental evidence that the ${ }^{90 \mathrm{Sr}} /{ }^{137} \mathrm{Cs}$ activity release ratio is constant and equal to 1.5 , allowing using the global determination of ${ }^{90} \mathrm{Sr}$ to estimate the ${ }^{137} \mathrm{Cs}$ one. Through it should be considered that once the nuclides are incorporated in the soil, the migration rates are different due to the dissimilar soil-nuclide interaction process. Since the wind circulation is presented in latitudinal bands, this is the assumption used to evaluate the transport and deposition of nuclides [UNSCEAR, 1982; UNSCEAR, 2000; UNSCEAR, 2008].

In order to compare the inventory data with the UNSCEAR predictions [UNSCEAR, 2000; UNSCEAR, 2008], in the Table 5 are presented the inventory of ${ }^{137}$ Cs data corrected by nuclide decay using the time of determination (a single input at 1965 is considered). It is observed that the experimental data do not follow the UNSCEAR prediction. The Fig. 8 shows the inventories of ${ }^{137} \mathrm{Cs}$, the average annual precipitation vs. latitude and the inventory vs. precipitation. Globally, it seems that the inventory depends on the annual precipitation and the Andes Cordillera plays a very important role on the inventory due to the generation of higher annual precipitations, hence more ${ }^{137} \mathrm{Cs}$ deposition. It is also observed that mountains act as barrier for Argentina.

\begin{tabular}{|c|c|c|c|}
\hline Code & $\begin{array}{c}{ }^{137} \text { Cs inventory } \\
\left(\mathrm{Bq} / \mathrm{m}^{2}\right)\end{array}$ & $\begin{array}{c}\text { Latitudinal band } \\
(\text { degree })\end{array}$ & $\begin{array}{c}\text { Integrated deposit } \\
\left(\mathrm{Bq} / \mathrm{m}^{2}\right)\end{array}$ \\
\hline BS6 & $945 \pm 110$ & \multirow{2}{*}{$0-10$} & 720 \\
\hline BS7 & $0.8 \pm 0.1$ & & \\
\hline BS8 & $5.15 \pm 0.07$ & & \\
\hline BS9 & $99 \pm 13$ & & \\
\hline BS10 & $83 \pm 11$ & & \\
\hline BS11 & $188 \pm 27$ & \\
\hline BS12 & $558 \pm 71$ & & \\
\hline BS13 & $654 \pm 212$ & \\
\hline BS14 & $16 \pm 2$ & & \\
\hline BS17 & $1120 \pm 76$ & & \\
\hline BS16 & $596 \pm 143$ & & \\
\hline BS15 & $1691 \pm 216$ & & \\
\hline BS4 & $621 \pm 46$ & & \\
\hline BS20 & $3494 \pm 477$ & & \\
\hline BS18 & $1333 \pm 79$ & & \\
\hline BS19 & $479 \pm 61$ & & \\
\hline BS5 & $594 \pm 37$ & & \\
\hline BS2 & $771 \pm 34$ & & \\
\hline BS3 & $614 \pm 77$ & & \\
\hline
\end{tabular}


Radiological Survey in Soil of South America

\begin{tabular}{|c|c|c|c|}
\hline Code & $\begin{array}{c}{ }^{137} \mathrm{Cs} \text { inventory } \\
\left(\mathrm{Bq} / \mathrm{m}^{2}\right)\end{array}$ & $\begin{array}{l}\text { Latitudinal band } \\
\text { (degree) }\end{array}$ & $\begin{array}{l}\text { Integrated deposit } \\
\left(\mathrm{Bq} / \mathrm{m}^{2}\right)\end{array}$ \\
\hline BS22 & $1355 \pm 173$ & & \\
\hline BS21 & $1407 \pm 180$ & & \\
\hline BS23 & $1375 \pm 176$ & & \\
\hline BS24 & $320 \pm 41$ & & \\
\hline BS25 & $3629 \pm 463$ & & \\
\hline BS26 & $1344 \pm 172$ & \multirow{30}{*}{$30-40$} & \multirow{30}{*}{1140} \\
\hline AS20 & 1877 & & \\
\hline AS21 & 1285 & & \\
\hline AS22 & 848 & & \\
\hline AS16 & 1311 & & \\
\hline AS17 & 1645 & & \\
\hline AS18 & 1427 & & \\
\hline AS19 & 745 & & \\
\hline AS12 & 2512 & & \\
\hline AS13 & 2041 & & \\
\hline AS14 & 2350 & & \\
\hline AS15 & 2050 & & \\
\hline AS4 & $689 \pm 36$ & & \\
\hline AS3 & $849 \pm 38$ & & \\
\hline AS1 & $950 \pm 34$ & & \\
\hline AS2 & $1366 \pm 38$ & & \\
\hline CS2 & 1438 & & \\
\hline CS3 & 2821 & & \\
\hline CS1 & 2202 & & \\
\hline CS5 & 1329 & & \\
\hline CS6 & 910 & & \\
\hline CS7 & 874 & & \\
\hline CS9 & 110 & & \\
\hline CS13 & 1565 & & \\
\hline CS16 & 1511 & & \\
\hline CS24 & 2111 & & \\
\hline CS25 & 2020 & & \\
\hline CS26 & 2584 & & \\
\hline CS27 & 2894 & & \\
\hline CS28 & 1893 & & \\
\hline
\end{tabular}




\begin{tabular}{|c|c|c|c|}
\hline Code & $\begin{array}{c}{ }^{137} \text { Cs inventory } \\
\left(\mathrm{Bq} / \mathrm{m}^{2}\right)\end{array}$ & $\begin{array}{c}\text { Latitudinal band } \\
\text { (degree) }\end{array}$ & $\begin{array}{c}\text { Integrated deposit } \\
\left(\mathrm{Bq} / \mathrm{m}^{2}\right)\end{array}$ \\
\hline CS29 & 3913 & & \\
\hline CS4 & 1420 & \multirow{18}{*}{$40-50$} & \multirow{18}{*}{1335} \\
\hline CS8 & 819 & & \\
\hline CS10 & 1128 & & \\
\hline CS11 & 1292 & & \\
\hline CS12 & 1492 & & \\
\hline CS14 & 1165 & & \\
\hline CS15 & 1438 & & \\
\hline CS17 & 892 & & \\
\hline CS18 & 2002 & & \\
\hline CS19 & 1238 & & \\
\hline CS20 & 2038 & & \\
\hline CS21 & 3130 & & \\
\hline CS22 & 1711 & & \\
\hline CS23 & 1674 & & \\
\hline CS30 & 2621 & & \\
\hline CS31 & 9045 & & \\
\hline CS32 & 4641 & & \\
\hline CS33 & 9846 & & \\
\hline CS34 & $1162 \pm 89$ & \multirow{14}{*}{$50-60$} & \multirow{14}{*}{705} \\
\hline CS35 & $1464 \pm 113$ & & \\
\hline CS36 & $1457 \pm 112$ & & \\
\hline CS37 & $1334 \pm 103$ & & \\
\hline CS45 & $796 \pm 61$ & & \\
\hline CS39 & $647 \pm 50$ & & \\
\hline CS38 & $630 \pm 48$ & & \\
\hline CS43 & $511 \pm 39$ & & \\
\hline CS40 & $527 \pm 41$ & & \\
\hline CS44 & $564 \pm 43$ & & \\
\hline CS46 & $991 \pm 76$ & & \\
\hline CS41 & $433 \pm 33$ & & \\
\hline CS42 & $546 \pm 42$ & & \\
\hline CS47 & $1673 \pm 129$ & & \\
\hline
\end{tabular}

Table 5. Determined ${ }^{137} \mathrm{Cs}$ inventory together with the UNSCEAR predictions [UNSCEAR, 2008], ordered by latitudinal band. 



Fig. 8. a) ${ }^{137}$ Cs inventory vs. annual precipitation; b) Annual precipitation vs. south latitude and c) ${ }^{137} \mathrm{Cs}$ inventory vs. annual precipitation. 


\subsection{Soil profile analysis}

The basic processes controlling mobility of anthropogenic nuclides in soil include convective transport by water, dispersion caused by spatial variations of convection velocities, diffusive movement within the fluid, and physicochemical interaction with soil matrix. Because the slow migration velocities of Cs in soils, generally the models do not take into account the soil moisture changes in the unsaturated zone but assume mean constant water content. The spatial uniformity of the deposition rates is also considered which may be defensible for the weapon tests and, at least, on a local scale in nuclear accidents. In this frame, the two trendy for modelling the migration of radionuclides in soils are the one dimensional convectiondispersion equation (ODCDE) with constant parameters [Likar et al., 2001; Bossen \& Kirchner, 2004] and the serial compartmental approach (CA) [Kirchner, 1998; Schuller et al., 1997].

The ODCDE model is based on the diffusive-convective transport, the mass conservation and the Cs-soil matrix interaction. The equation describing the migration process is usually known as the Fokker Planck equation:

$$
\frac{\partial C(x, t)}{\partial t}=D_{e} \frac{\partial^{2} C(x, t)}{\partial^{2} x}-v_{e} \frac{\partial C(x, t)}{\partial x}-\lambda C(x, t)
$$

where $C(x, t)$ is the ${ }^{137} C$ s concentration in the soil (mobile and sorbed), $\lambda$ is the decay constant, $D_{e}$ is the effective diffusion coefficient of caesium in soil, $v_{e}$ is a convective velocity, $x$ is the soil depth with respect to the soil surface and $t$ is the time from the deposition. Assuming that all sorbed $\mathrm{Cs}$ is exchangeable and that the exchange process is in equilibrium, $D_{e}$ is an effective coefficient that depends on the porosity $\varepsilon$, the bulk density $\rho$ and the solid aqueous partitioning coefficient $K_{d}$ :

$$
D_{e}=\frac{D_{w}}{\left(1+\rho K_{d} / \varepsilon\right)} \quad v_{e}=\frac{v_{w}}{\left(1+\rho K_{d} / \varepsilon\right)}
$$

and where $D_{w}$ and $v_{w}$ are the diffusion coefficient of Cs in soil water and the convection velocity of water in pores of soil, respectively. The factor between parentheses in eqs. 3 is called the retardation factor. As boundary conditions, a half-infinite space-time is assumed and the considered initial condition is a pulse-like deposit at $t=0$ with deposition density $J_{0}$. With all these assumptions, it is obtained the well known solution:

$$
C(x, t)=J_{0} e^{-\lambda t}\left[\frac{1}{\sqrt{\pi D_{e} t}} e^{-\left(x-v_{e} t\right)^{2} / 4 D_{e} t}-\frac{v_{e}}{2 D_{e}} e^{-v_{e} / D_{e}} \operatorname{erfc}\left(\frac{v_{e}}{2} \sqrt{\frac{t}{D_{e}}+\frac{x}{2 \sqrt{D_{e}} t}}\right] .\right.
$$

The irreversible fixation of Cs to soil has been also accounted for, however in this case, no analytical solution of the transport equations are able to obtain, so numerical methods are needed to obtain the concentration profiles [Antonopoulus-Domis et al., 1995; Toso \& Velasco, 2001]. The fitting of experimental data with the ODCDE model allows determining $D_{e}$ and $v_{e}$.

The CA has been used to analyse the depth layered profiles without detailed information about the site-specific processes that influence radionuclide's mobility. Usually the soil profile is split into a series of horizontal layers (compartments) which are connected by 
radionuclide downward transport rates and the migration dynamic is described by a system of lineal first-order differential equations with constant coefficients. This model is applicable only if the transport of the radionuclide is dominated by convection [Kirchner et al., 2009]. It is worth to mention that neither the presence of micro-organisms nor the root intake is considered by the models.

In the following, data compilation of the transport parameters of soils of South America is presented in Fig. 9. To facilitate the comparison, the worldwide average values corresponding to weapons fallout have been also included [IAEA, 2010]. In the La Plata city area of the Buenos Aires Province, the two profiles (AS1 and AS3) that clearly have a Gaussian shape were fitted using the ODCDE [Montes et al., 2010a; Montes et al., 2010b]. For the AS1, the $D_{e}$ and $v_{e}$ resulted equal to $0.728 \mathrm{~cm}^{2} / \mathrm{y}$ and $0.23 \mathrm{~cm} / \mathrm{y}$, respectively. while for AS3 the values were $0.5 \mathrm{~cm}^{2} / \mathrm{y}$ and $0.22 \mathrm{~cm} / \mathrm{y}$, correspondingly. Since in the case of profile of AS2 site, the Gaussian-shape was established down to $7 \mathrm{~cm}$ in depth, the data of the top layer were disregarded in the analysis leading to diffusion coefficient and convection velocity values of $0.39 \mathrm{~cm}^{2} / \mathrm{y}$ and $0.34 \mathrm{~cm} / \mathrm{y}$, respectively. These set of transport parameter values agrees well with the South American values [Juri Ayub et al., 2007; Juri Ayub et al., 2008; Schuller et al., 1997; Schuller et al., 2004], as observed in Fig. 6, but is slightly larger than the average values reported by IAEA [IAEA, 2010].

In the semi-natural and natural central area of Argentina (AS16-AS24), the diffusion coefficients obtained using the ODCDE varied from $0.43 \mathrm{~cm}^{2} / \mathrm{y}$ to $2.27 \mathrm{~cm}^{2} / \mathrm{y}$, and the convection velocity varied from $0.13 \mathrm{~cm} / \mathrm{y}$ to $0.39 \mathrm{~cm} / \mathrm{y}$. The $D_{e}$ values were in the range reported in the bibliography for some Chilean and European soils, while the $v_{e}$ values were one order of magnitude higher than those reported for Chilean soils and of the same order of magnitude than the European sandy ones [IAEA, 2010]. The great penetration in these soils was ascribed to the high sand and low fine materials content, i.e., high porosity facilitating water passage to deeper layers.

The Chilean Southern soil profiles of these soils were analysed using both, the CA and the ODCDE. The results of the fits were not good. In the case of the CA, the variation of the migration rates did not improve appreciably the fits. Moreover, the CS3 profile cannot be reproduced with this model. The determined migration rates resulted always low, between $0.1 \mathrm{~cm} / \mathrm{y}$ and $0.3 \mathrm{~cm} / \mathrm{y}$, in the lower range of the reported data obtained for nuclear weapons and Chernobyl fallout [Schuller et al., 1997]. On the other side, the analysis of the data using the ODCDE indicated that the transport was dominated by diffusion process in agreement with the high silt and clay content of the studied soils. The agreement of $D_{e}$ and $v_{e}$ with reported data was better than in the CA case. However some misfit was observed at large depth, probably due to preferential transport through macropores, migration of suspended particles, spatial variability or agricultural land disturbance. Least-square fits of semi-natural and natural South Patagonia profiles using the ODCDE with constant parameters and improved by assuming a logarithmic distribution of $D_{e}$ and $v_{e}$ or depth dependence of both parameters were also tried. In all attempts, the mean obtained parameters were the same. The convection velocity was found to be negative (upward migration) in the CS43 site, because of the yearly flooded lowland, and at CS45 soil, where the upper soil layer was possibly disturbed by animal hoof prints. In the CS43 site, the $D_{e}$ values were considerably larger than the determined for the other profiles. In the other sites, the determined median convection velocity and the diffusion coefficient values were $0.056 \mathrm{~cm} / \mathrm{y}$ and $0.048 \mathrm{~cm}^{2} / \mathrm{y}$, respectively. The 
convection velocities resulted rather higher when compared with the data of temperate regions from Chile [Schuller et al., 1997], while the diffusion coefficient was close to those obtained in the Antarctic region [Schuller et al., 2002].

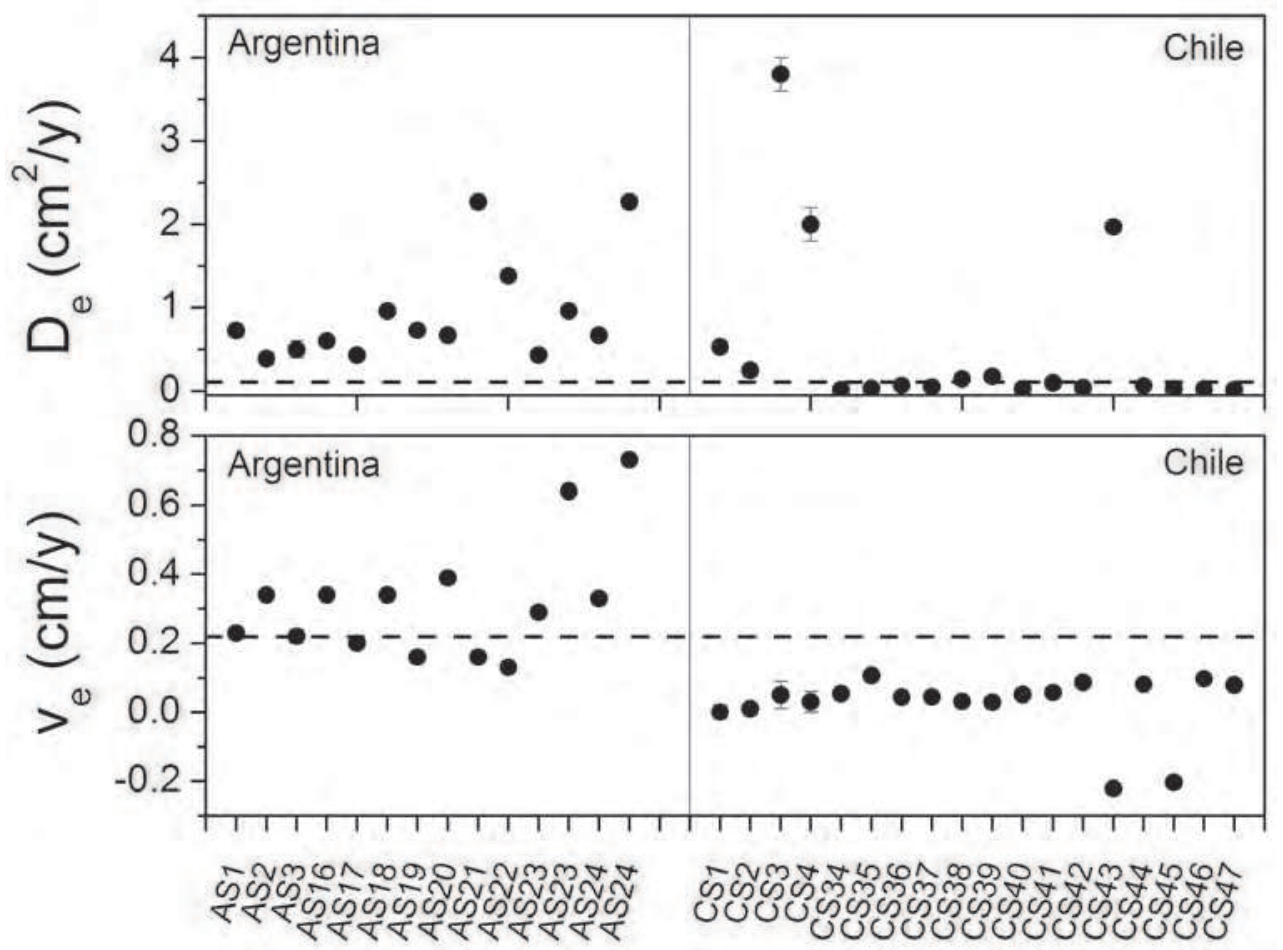

Fig. 9. Diffusion coefficient $\left(D_{e}\right)$ and convection velocity $\left(v_{e}\right)$ parameters together with the IAEA (dash line) [IAEA, 2010].

The present set of transport parameters of ${ }^{137} \mathrm{Cs}$ in soils is presented in Fig. 9. A scatter is observed in the data. Most of Argentinean diffusion coefficient data are slightly larger than the average values reported by IAEA for weapon test fallout [IAEA, 2010] while the Chilean data fit quite well with the average values with the exception of CS3, CS4 and CS43. Concerning the convection velocity parameter, the determined values from Provincia de Buenos Aires-Argentina soils resulted close to the worldwide average ones [IAEA, 2010], while those determined in the San Luis Province-Argentina are higher than the IAEA data [IAEA, 2010]. In Chile, the values were lower than the worldwide average [IAEA, 2010], being some of them negative, probably related to the periodic flooded lowland. Several mechanisms, such as bioturbation, horizontal transport, transport through macropores, migration of suspended particles, etc., have been used to explain the deviations from the convection-dispersion predictions, putting the ODCDE model under consideration and suggesting that the model is an oversimplification of such a complex process. However the ODCDE model is very useful to estimate the transport parameters. 


\section{Conclusions}

A systematic compilation of radionuclide activity data in soil of South America has been completed. Radionuclide activity data concern to the natural ${ }^{40} \mathrm{~K},{ }^{238} \mathrm{U}$ and ${ }^{232} \mathrm{Th}$ chains, and to the anthropogenic ${ }^{137} \mathrm{Cs}$ nuclides.

The surface activity concentrations for ${ }^{238} \mathrm{U}$ for Brazil and Argentina are higher that the worldwide mean values. The $232 \mathrm{Th}$ activity data of Argentina are closer to the worldwide values while the Brazilian ones are quite higher than the worldwide values. In the case of Uruguay, it is not possible to extract conclusions yet due to the insufficiency and dispersion of data. The ${ }^{40} \mathrm{~K}$ data are higher than the mean values in most of the cases, and fit into the worldwide range with some exceptions. The annual committed effective terrestrial exposure dose for infants, children and adults have been calculated, resulting the values slightly higher than the UNSCEAR value in the case of adults. The analysis of the ${ }^{137} \mathrm{Cs}$ inventories allows concluding that the experimental data do not follow the latitudinal band deposition predictions proposed by UNSCEAR. It is worth to mention that the analysis of the whole set of information in South America allows to establish a correlation between the inventory and the annual precipitations. Different shape type profiles have been determined for Argentina, Brazil and Chile. In several cases it was possible to reproduce the ${ }^{137} \mathrm{Cs}$ profiles with models accounting diffusion and convection process. The transport parameters agree well with the average worldwide values due to nuclear weapon test fallout. Some discrepancies were detected when bioturbation and floodedland are present, indicating that efforts to include these processes should be done to fully reproduce the caesium profiles, hence to be able to make predictions of migration in case of possible pollution.

The present set of data contributes to the establishment of regional baselines as well as help in the development of local regulations concerning to permitted activity limits to people health protection.

\section{Acknowledgements}

Research grants PIP 0230 from Consejo Nacional de Investigaciones Científicas y Técnicas (CONICET, Argentina) and PICT 38047-Préstamo BID from Agencia de Promoción Científica (ANCYT, Argentina) are gratefully recognized.

\section{References}

Antonopoulus-Domis, M.; Clouvas, A.; Hiladakis, A. \& Kadi, S. (1995). Radiocesium distribution in undisturbed soil: Measurements and diffusion-advection model. Health Physics, Vol.69, pp. 949-953

Bellenber, J. P. \& Stauton, S. (2008). Adsorption and desorption of ${ }^{85} \mathrm{Sr}$ and ${ }^{137} \mathrm{Cs}$ on reference minerals, with and without inorganic and organic surface coatings. Journal of Environmental Radioactivity, Vol. 99, pp. 831-840, ISSN 0265 931X

Bossen, P. \& Kirchner, G. (2004). Modeling the vertical distribution of radionuclides in soils. Part I: the convection-dispersion equation revisited. Journal of Environmental Radioactivity, Vol. 73, pp. 127-150, ISSN 0265 931X

Bujan, A.; Santanatoglia, O. J.; Chagas, C.; Massobrio, M.; Castiglioni, M.; Yañez, M.; Ciallella, H. \& Fernandez, J. (2000). Preliminary study on the use of the ${ }^{137} \mathrm{Cs}$ 
method for soil erosion investigation in the pampean region of Argentina. Acta Geologica Hispanica, Vol. 35, pp. 271-277, ISSN 16956133

Bujan, A.; Santanatoglia, O. J.; Chagas, C.; Massobrio, M.; Castiglioni, M.; Yañez, M.; Ciallella, H. \& Fernandez, J. (2003). Soil erosion evaluation in a small basing though the use of ${ }^{137} \mathrm{Cs}$ technique. Soil $\mathcal{E}$ Tillage Research, Vol. 69, pp. 127-137, ISSN 0167 1987

Catallette, H.; Dumonceau, J. \& Ollar, P. (1998). Sorption of cesium, barium and europium on magnetite. Journal of Contaminant Hydrology, Vol. 35, pp. 151-159, ISNN 01697722

Cooper, J. R.; Randle \& K.; Sokhi, R. S. (2003). In: Radioactive Releases in the Environment, John Wiley \& Sons Ltd. ISBN 047189924 0, The Atrium, Southern Gate, Chichester, West Sussex, England

Cornell, R. M. (1993). Adsorption of cesium on minerals: a review. Journal of Radioanalytical and Nuclear Chemistry, Vol. 171, pp. 483 - 500. ISNN 02365731

Correchel, V.; Oliveira Santos Bacchi, O.; Reichardt, K. \& Cereci de Maria I. (2005). Random and systematic spatial variability of ${ }^{137} \mathrm{Cs}$ inventories at references sites in southcentral Brazil. Scientia Agricola, Vol. 62, pp. 173-178, ISSN 01039016

Handl, J.; Sachase, R.; Jakob, D.; Michel, R.; Evangelista, H.; Gonçalves, A. C. \& de Freitas, A. C. (2008). Accumulation of ${ }^{137} \mathrm{Cs}$ in Brazilian soils and its transfer to plants under different climatic conditions. Journal of Environmental Radioactivity, Vol. 99, pp. 271287, ISSN $0265931 X$

IAEA. (2010). Handbook of Parameter Values For The Prediction Of Radionuclide Transfer In Terrestrial And Freshwater Environments. Technical Reports Series No. 472, Vienna.

Imbellone, P. A.; Guichon, B. A. \& Giménez, J. E. (2009). Hydromorphic soils of the River Plate coastal plain, Argentina. Latin american Journal of sedimentology and basin analysis, Vol. 16, pp. 3-18, ISNN 1669-73616

Juri Ayub, J.; Rizzotto, M.; Toso, J. \& Velasco, H. (2007). ${ }^{137}$ Cs deposition and vertical migration in soils from Argentina. Proc. International. Conf. on Environ. Radioctivity: From Measurements and Assessments to Regulation, Vienna, Austria, www-pub.iaea.org/mtcd/meetings/announcements.asp?confid=145

Juri Ayub, J.; Velasco, R.H.; Rizzotto, M.; Quintana E. \& Aguiar J. (2008). ${ }^{40 K}$, ${ }^{137 C s}$ and $226 \mathrm{Ra}$ soil and plant content in semi-natural grasslands of Central Argentina. The Natural Radiation Environment-8 $8^{\text {th }}$ International Symposium, edited by A. S. Paschoa, American Institute of Physics, ISBN 978073540559

Kirchner, G. (1998). Applicability of compartmental models for simulating the transport of radionuclides in soil. Journal of Environmental Radioactivity, Vol. 38, pp. 339-352, ISSN 0265 931X

Kirchner, G.; Strebl, F.; Bossew, P.; Ehlken, S. \& Gerzabek, H. (2009). Vertical migration of radionuclides in undisturbed grassland soils. Journal of Environmental Radioactivity, Vol. 100, pp. 716-720, ISSN 0265 931X

Malanca, A.; Gaidolfi, L.; Pessina, V.\& Dallara G. (1996). Distribution of ${ }^{226 R a,}{ }^{232} \mathrm{Th}$, and ${ }^{40 K}$ in soils of Rio Grande do Norte (Brazil). Journal of Environmental Radioactivity, Vol. 30, pp. 55-67, ISSN $0265931 \mathrm{X}$ 
Marmier, N. \& Fromage, F. (2000). Sorption of Cs(I) on Magnetite in the Presence of Silicates. Journal of Colloid and Interface Sciencie, Vol. 223, pp. 83-88

Montes, M. L.; Taylor, M. A.; Mercader, R. C.; Sives, F. R. \& Desimoni, J. (2010a). Hyperfine and radiological characterization of soils of the province of Buenos Aires. Argentina. Journal of Physics: Conference Series, Vol. 217, pp. 012058 012062, ISSN 17426596

Montes, M. L.; Mercader, R. C.; Taylor, M. A.; Runco, J.; Ibellone, P.A.; Rivas, P. C. \& Desimoni, J. (2010b). Radiological and Hyperfine Characterization of soils from the Northeastern region of the Province of Buenos Aires, Argentina. Hyperfine Interactions. Submitted, ISSN 03043834

Montes, M. L. (2011). Private communication

Odino Moure, M. R. (2010). Environmental Radioactivity Monitoring Plan in Uruguay. Technical Meeting on in-situ methods for characterization of contaminated sites. Vienna, Austria. http://www-pub.iaea.org/mtcd/meetings/Announcements.asp?ConfID=38924

Sajó-Bohus, L.; Pálfalvi, J.; Urbani, F.; Castro, D.; Greaves, E.D.\& Liendo, J.A. (1999). Environmental gamma and radon dosimetry in Venezuela. Radiation Measurements, Vol. 31, pp. 283-286, ISSN 13504487

Salbu, B. (2009). Fractionation of radionuclide species in the environment. Journal of Environmental Radioactivity, Vol. 100, pp. 283-289, ISSN 0265 931X

Sawhney, B. L. (1972). Selective sorption and fixation of cations by clay minerals: A review. Clays and Clay Minerals, Vol. 20, pp. 93-100, ISSN 0009-8604

Schuller, P.; Ellies, A. \& Kirchner, G. (1997). Vertical migration of fallout ${ }^{137}$ Cs in agricultural soils from southern Chile. Science of the Total Environment, Vol. 193, pp. 197-205, ISSN: 00489697

Schuller, P.; Voigt, G.; Handl, J.; Ellies, A. \& Oliva, L. (2002). Global weapons fallout ${ }^{137}$ Cs in soils and transfer to vegetation in south-central Chile. Journal of Environmental Radioactivity, Vol. 62, pp. 181-193, ISSN 0265 931X

Schuller, P.; Bunzl, K.; Voigt, G.; Ellies, A. \& Castillo, A. (2004). Global fallout 137 Cs accumulation and vertical migration in selected soils from south Patagonia, Journal of Environmental Radioactivity, Vol. 71, pp. 43-60, ISSN 0265 931X

Singh, B. K.; Jain, A.; Kumar, S.; Tomar, B. S.; Tomar, R.; Manchanda, V. K. \& Ramanathan, S. (2009). Role of magnetite and humic acid in radionuclide migration in the environment, Journal of Contaminant Hydrology, Vol. 106, pp. 144-149. ISNN 01697722

Staunton, S.; Dumat, C.; \& Zsolnay, A. (2002). Possible role of organic matter in radiocaesium adsorption in soils. Journal of Environmental Radioactivity, Vol. 58, pp. 163-173, ISSN $0265931 X$

Tomazini da Conceic, F. \& Bonotto, D.M. (2006). Radionuclides, heavy metals and fluorine incidence at Tapira phosphate rocks, Brazil, and their industrial products. Environmental Pollution, Vol. 139, pp. 232-243, ISSN 0269-7491 
Toso, J. P. \&Velasco, R.H. (2001). Describing the observed vertical transport of radiocesium in specific soils with three time-dependent models. Journal of Environmental Radioactivity, Vol. 53, pp. 133-144, ISSN 0265 931X

UNSCEAR, (1982). In: Ionizing Radiation: Sources and Biological effects, Report of the General Assembly with Scientific Annexes, Vol. 1, Annex E, New York,

UNSCEAR, (2000). In: Sources and effects of ionizing radiation, Report of the General Assembly with Scientific Annexes, Vol. 1, New York

UNSCEAR, (2008). In: Sources and effects of ionizing radiation, Report of the General Assembly with Scientific Annexes, Vol. 1, New York

Valdés, M. E.; Blanco, M. V.; Taylor, M. A.; Sives, F.R.;Runco, J. \& Desimoni J. (2011). Determinación de las actividades de ${ }^{60} \mathrm{Co},{ }^{137} \mathrm{Cs}$ y ${ }^{235} \mathrm{U}$ en muestras de suelo, sedimentos y agua provenientes de la zona aledaña a una instalación nuclear. Ciencia Forense Latinoamericana, in press.

Vandenberghe, R. E. (1991). In: Mössbauer Spectroscopy and Applications in Geology, International Training Centre for Post-graduate soil Scientists, Geological Institute, Faculteit der Wetenschappen, Faculty of Science, Belgium.

Velasco, H. R.; Jury. Ayub, J.; Belli, M.; \& Sansone, U. 2006. Interaction matrices as a first step toward a general model of radionuclide cycling: Application to the ${ }^{137} \mathrm{Cs}$ behaviour in a grassland ecosystem. Journal of Radioanalytical and Nuclear Chemistry, Vol. 268, pp. 503-509. ISSN 02365731

Valkovic, V. (2000). In: Radioactivity in the Environment, Elsevier, ISBN-13: 9780444829542. 


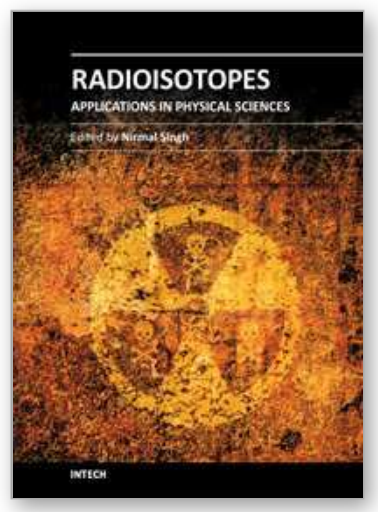

\author{
Radioisotopes - Applications in Physical Sciences \\ Edited by Prof. Nirmal Singh
}

ISBN 978-953-307-510-5

Hard cover, 496 pages

Publisher InTech

Published online 19, October, 2011

Published in print edition October, 2011

The book Radioisotopes - Applications in Physical Sciences is divided into three sections namely:

Radioisotopes and Some Physical Aspects, Radioisotopes in Environment and Radioisotopes in Power System Space Applications. Section I contains nine chapters on radioisotopes and production and their various applications in some physical and chemical processes. In Section II, ten chapters on the applications of radioisotopes in environment have been added. The interesting articles related to soil, water, environmental dosimetry/tracer and composition analyzer etc. are worth reading. Section III has three chapters on the use of radioisotopes in power systems which generate electrical power by converting heat released from the nuclear decay of radioactive isotopes. The system has to be flown in space for space exploration and radioisotopes can be a good alternative for heat-to-electrical energy conversion. The reader will very much benefit from the chapters presented in this section.

\title{
How to reference
}

In order to correctly reference this scholarly work, feel free to copy and paste the following:

Maria Luciana Montes and Judith Desimoni (2011). Radiological Survey in Soil of South America,

Radioisotopes - Applications in Physical Sciences, Prof. Nirmal Singh (Ed.), ISBN: 978-953-307-510-5, InTech, Available from: http://www.intechopen.com/books/radioisotopes-applications-in-physical-sciences/radiologicalsurvey-in-soil-of-south-america

\section{INTECH}

open science | open minds

\author{
InTech Europe \\ University Campus STeP Ri \\ Slavka Krautzeka 83/A \\ 51000 Rijeka, Croatia \\ Phone: +385 (51) 770447 \\ Fax: +385 (51) 686166 \\ www.intechopen.com
}

\author{
InTech China \\ Unit 405, Office Block, Hotel Equatorial Shanghai \\ No.65, Yan An Road (West), Shanghai, 200040, China \\ 中国上海市延安西路65号上海国际贵都大饭店办公楼 405 单元 \\ Phone: +86-21-62489820 \\ Fax: $+86-21-62489821$
}


(C) 2011 The Author(s). Licensee IntechOpen. This is an open access article distributed under the terms of the Creative Commons Attribution 3.0 License, which permits unrestricted use, distribution, and reproduction in any medium, provided the original work is properly cited. 